Rafael Silva do Nascimento

\title{
Fossil Birds of Brazil
}

\author{
Aves Fósseis do Brasil
}

Single Volume 


\title{
Rafael Silva do Nascimento
}

\section{Fossil Birds of Brazil}

\author{
Aves Fósseis do Brasil
}

Single Volume

Simplified Version

\begin{abstract}
Dissertation submitted to the Graduate Program of the Museu de Zoologia da Universidade de São Paulo in partial fulfillment of the requirements for the degree of Master of Science (Systematics, Animal Taxonomy and Biodiversity).
\end{abstract}

Advisor: Prof. Dr. Luís Fábio Silveira 


\section{RESUMO}

A paleontologia de aves no Brasil é um campo de pesquisa ainda incipiente, a despeito de seu material relativamente abundante. Os vestígios são representados por ossos (fósseis e subfósseis), impressões de penas, ovos, coprólitos e icnofósseis, encontrados em todas as Regiões do país a partir da década de 1830. A revisão e a catalogação do histórico do estudo e de todos esses registros, com atualização de sua nomenclatura taxonômica, incluindo detalhamento do material, sinonímia, dados de deposição e inferências paleobiológicas, são importantes para fornecer um panorama de sua diversidade, além de oferecer subsídios para estudos filogenéticos, taxonômicos e biogeográficos. O levantamento da literatura resultou em 378 registros, representados pelos seguintes grupos: Maniraptora (penas) (70), Aves indet. (23), Columbidae (20), Psittacidae (18), Rallidae (17), Accipitridae (16), Tinamidae (12), Cathartidae (9), Falconidae (9), Passeriformes (9), Strigidae (9), Anhingidae (8), Picidae (8), Anatidae (7), Cracidae (7), Caprimulgidae (6), Ciconiidae (6), Procellaridae (5), Scolopacidae (5), Thamnophilidae (5), Thraupidae (5), Trochilidae (5), Cuculidae (4), Dendrocolaptidae (4), Enantiornithes (4), Icteridae (4), Podicipedidae (4), Tyrannidae (4), Cariamiformes (3), Hirundinidae (3), Laridae (3), Phalacrocoracidae (3), Phorusrhacidae (3), Rheidae (3), Sulidae (3), Ardeidae (2), Bucconidae (2), Charadriidae (2), Diomedeidae (2), Formicariidae (2), Furnariidae (2), Momotidae (2), Nyctibiidae (2), Palaeognathae (2), Quercymegapodiidae (2), Ramphastidae (2), Threskiornithidae (2), Troglodytidae (2), Trogonidae (2), Turdidae (2), Vireonidae (2), Alcenididae (1), Anhimidae (1), Apodidae (1), Cardinalidae (1), Cariamidae (1), Corvidae (1), Fregatidae (1), Gracilitarsidae (1), Jacanidae (1), Mimidae (1), Neognathae (1), Odontophoridae (1), Opisthocomidae (1), Ornithuromorpha (1), Palaelodidae (1), Passerellidae (1), Pelagornithidae (1), Phoenicopteridae (1), Spheniscidae (1), Strigiformes (1), Teratornithidae (1) e Tytonidae (1). Dentre esse número, foram descritos 16 gêneros e 21 espécies extintos. A maior parte do material foi encontrada em cavernas calcárias com idade quaternária, quase que exclusivamente representada por neoespécies. No geral, uma grande quantidade de material aguarda análises mais aprofundadas, incluindo elementos não diagnósticos, não acessíveis ou podendo representar neoespécies no caso de material mais recente.

Palavras-chave: Ornitologia. Paleontologia. Arqueologia. História da ciência. América do Sul. 


\section{ABSTRACT}

Avian paleontology in Brazil is a research field still in its early stages despite its relatively abundant material. The remains are represented by bones, feather impressions, eggs, coprolites, and ichnofossils found in all Regions of Brazil starting in the 1830s. Reviewing and cataloging the history of studies and all records, with updates of their taxonomic nomenclature, including detailed material, synonyms, data on deposition, and paleobiological inference, are important to provide an overview of their diversity and offer subsidies for phylogenetic, taxonomic, and biogeographic studies. The literature survey resulted in 378 records represented by the following groups (number of taxa in brackets Maniraptora (penas) (70), Aves indet. (23), Columbidae (20), Psittacidae (18), Rallidae (17), Accipitridae (16), Tinamidae (12), Cathartidae (9), Falconidae (9), Passeriformes (9), Strigidae (9), Anhingidae (8), Picidae (8), Anatidae (7), Cracidae (7), Caprimulgidae (6), Ciconiidae (6), Procellaridae (5), Scolopacidae (5), Thamnophilidae (5), Thraupidae (5), Trochilidae (5), Cuculidae (4), Dendrocolaptidae (4), Enantiornithes (4), Icteridae (4), Podicipedidae (4), Tyrannidae (4), Cariamiformes (3), Hirundinidae (3), Laridae (3), Phalacrocoracidae (3), Phorusrhacidae (3), Rheidae (3), Sulidae (3), Ardeidae (2), Bucconidae (2), Charadriidae (2), Diomedeidae (2), Formicariidae (2), Furnariidae (2), Momotidae (2), Nyctibiidae (2), Palaeognathae (2), Quercymegapodiidae (2), Ramphastidae (2), Threskiornithidae (2), Troglodytidae (2), Trogonidae (2), Turdidae (2), Vireonidae (2), Alcenididae (1), Anhimidae (1), Apodidae (1), Cardinalidae (1), Cariamidae (1), Corvidae (1), Fregatidae (1), Gracilitarsidae (1), Jacanidae (1), Mimidae (1), Neognathae (1), Odontophoridae (1), Opisthocomidae (1), Ornithuromorpha (1), Palaelodidae (1), Passerellidae (1), Pelagornithidae (1), Phoenicopteridae (1), Spheniscidae (1), Strigiformes (1), Teratornithidae (1), and Tytonidae (1). Among these, 16 extinct genera and 21 extinct species were described. Most of the material was found in limestone caves of quaternary age and represents almost exclusively neospecies. In general, a large quantity of material awaits deeper analyses, including elements that are non-diagnostic, non-accessible or that may represent neospecies in the case of more recent material.

Keywords: Ornithology. Paleontology. Archeology. History of science. South America. 


\section{INTRODUCTION}

Brazil currently has 1,971 recognized bird species (Pacheco et al. 2021), being the country with the most expressive diversity in the world. This diversity corresponds to the geological moment between the Holocene and the Anthropocene and represents a very small fraction of the time of existence of birds. In a country of continental dimensions such as Brazil, it is reasonable to assume that the number of species that existed overtime in its territory is equally significant, comprising an enormous diversity of shapes, sizes, colors, and habits that, even in a fortunate situation, will have only a relatively small portion known from the fossil record.

While the living species in Brazil are particularly well studied (Sick 1997), knowledge on their prehistoric representatives has been progressing slowly, especially when compared to countries like Germany, China, the United States, and Argentina, the last of which holds the most complete and diverse record in South America. The fossil record of birds is notably biased, especially when compared to mammals, due to the fragile nature of their bones (Agnolin 2016b). In addition, the small number of specialized researchers working in the field and the lack of osteological collections hinder, among other factors, the development of both paleornithology and systematics in Brazil (Alvarenga 1992). Despite this, in the last decades, we witnessed 21 extinct species being formally described from paleontological sites across the country, with the oldest records dating back to the early Cretaceous (Winge 1887; Ameghino 1891; Alvarenga 1982; 1983; 1985a; 1985b; 1988; 1990; 1995; 1999; Baird \& Vickers-Rich 1997; Olson \& Alvarenga 2002; Alvarenga \& Guilherme 2003; Alvarenga \& Olson 2004; Alvarenga et al. 2008; Faure et al. 2010; Mayr et al. 2011a; Mayr et al. 2011b; Carvalho et al. 2015; 2021). Material of Quaternary age was found in considerable number, such as the records from Bahia (e.g., Penido et al. 2012), Piauí (e.g., Guérin et al. 1996), and Minas Gerais (e.g., Souza Cunha \& Guimarães 1978), but relatively little has been published on it.

The avian remains found in the country are represented by bones, feathers, mummified specimens, eggs, coprolites, pellets, and alleged tracks (e.g., Castro et al. 1988; Alvarenga 1997; Figuti 2005; Lopes et al. 2011; Marsola et al. 2014) and are housed in museums or private collections in Brazil, Denmark, the United States, 
England, France, Germany, and Japan (e.g., Reinhardt 1881; Winge 1887; Ameghino 1891; Olson 1981; Alvarenga 1982; Naish et al. 2007). In addition, rock paintings made by the ancient human inhabitants of the territory also testify to their presence (Sick 1997).

Published studies are focused almost entirely on taxonomy (Taranto 2012: 24). Most of the Brazilian material determined so far belongs to extant species, and yet its attribution to specific taxa cannot be accepted without a doubt (Olson 1985). Rarely have older studies been considered in more recent ones, and several mentions are limited to lists of taxa without specifying the state and nature of the material. Some studies are even overlooked due to being published in volumes focused on other areas of prehistory's knowledge.

The lack of comparative material is a significant hindrance to the determination of the fossils, becoming even more critical when treating materials from older geological periods. Referring to younger material from caves, Sales (2003: 212) noted that, besides the rare preservation of bird remains in these environments, which disappear before the calcification process, this material's location is also compromised by the lack of knowledge and records of Brazilian caves, added to the scarcity of biologists and speleologists working in this line of research. In Brazil, there is virtually no specialist dedicated exclusively to the study of fossil birds, a scenario prompted by several factors that include nationally generalized poor valorization of science to possibly the idea that birds do not fossilize well and, therefore, there are no materials of this group to be studied intending a full-time dedication. There are several mentions of bird material, generally regarded as indeterminate or referred to as neospecies, in conference abstracts by undergraduate students, and, in rare exceptions, this line of research is carried on in their careers. Most of the recent bird material described in full articles is done by researchers who also cover other groups (generally vertebrates) in their publications.

Therefore, it is necessary to organize this fragmented information on Brazilian paleornithology and start new studies to expand its components. Besides broadening the understanding of the taxonomic diversity and the evolution of birds, such knowledge is 
an essential source of paleobiogeographic and paleoenvironmental information (Alvarenga \& Höfling 2000: 590).

\section{CONCLUSION}

A complete panorama of the avian fossil and subfossil diversity in Brazil is far from being reached, but herein we present a list with the most important records present in the literature. This present list is naturally biased by several factors of biological, geological, and political nature, but, to date, this is the most complete picture of Brazilian paleornithogical studies ever compilated.

In general, the most complete fossil avifauna known for the country is the one from the Quaternary of Lagoa Santa Karst, which is also the first to be studied. The most complete from the Paleogene is that from Tremembé Formation. The most diverse known non-Quaternary avifauna is that from the Tremembé Formation. Of the 77 feather records, 70 are of Mesozoic age, all belonging to the Crato Formation, and cannot be assigned with security to avian dinosaur clades. Two fossil egg records have been published, with no ootaxa erected for them. Two ichnofossil records have been associated with birds, with one of them needing revision.

The following sections analyze this compilation from different points of view.

\section{1. Taxonomic representation}

The 378 taxa recorded can be divided into the following family or equivalent clades (from the most to the less numerous): Maniraptora (feathers) (70), Aves (23), Columbidae (20), Psittacidae (18), Rallidae (17), Accipitridae (16), Tinamidae (12), Cathartidae (9), Falconidae (9), Passeriformes (9), Strigidae (9), Anhingidae (8), Picidae (8), Anatidae (7), Cracidae (7), Caprimulgidae (6), Ciconiidae (6), Procellaridae (5), Scolopacidae (5), Thamnophilidae (5), Thraupidae (5), Trochilidae (5), Cuculidae (4), Dendrocolaptidae (4), Enantiornithes (4), Icteridae (4), Podicipedidae (4), Tyrannidae (4), Cariamiformes (3), Hirundinidae (3), Laridae (3), Phalacrocoracidae (3), Phorusrhacidae (3), Rheidae (3), Sulidae (3), Ardeidae (2), Bucconidae (2), Charadriidae (2), Diomedeidae (2), Formicariidae (2), Furnariidae (2), Momotidae (2), Nyctibiidae (2), Palaeognathae (2), Quercymegapodiidae (2), Ramphastidae (2), 
Threskiornithidae (2), Troglodytidae (2), Trogonidae (2), Turdidae (2), Vireonidae (2), Alcenididae (1), Anhimidae (1), Apodidae (1), Cardinalidae (1), Cariamidae (1), Corvidae (1), Fregatidae (1), Gracilitarsidae (1), Jacanidae (1), Mimidae (1), Neognathae (1), Odontophoridae (1), Opisthocomidae (1), Ornithuromorpha (1), Palaelodidae (1), Passerellidae (1), Pelagornithidae (1), Phoenicopteridae (1), Spheniscidae (1), Strigiformes (1), Teratornithidae (1), and Tytonidae (1). If we exclude Maniraptora and Aves, which comprises most indeterminate taxa, Columbidae is the clade with the best taxonomical representation in the fossil records of birds in Brazil.

Most records belong to living taxa (145). Extinct taxa are represented by 132 records, and 101 records do not have sufficient taxonomical determination to classify them into one of these categories. Two possibly recently, anthropically extinct taxa are present in the fossil record (Anodorhynchus glaucus and Paraclaravis geoffroyi), but here we considered them as living taxa.

The following orders of native Brazilian living avifauna (following Pacheco et al. 2021) do not have published fossil representatives found during this research: Steatornithiformes, Eurypygiformes, and Phaethontiformes. In the same category, the following families do not have fossil published fossil representatives: Steatornithidae, Aramidae, Psophiidae, Heliornithidae, Haematopodidae, Recurvirostridae, Burhinidae, Chionidae, Thinocoridae, Rostratulidae, Glareolidae, Stercorariidae, Eurypygidae, Phaethontidae, Oceanitidae, Hydrobatidae, Pelecanidae, Pandionidae, Galbulidae, Capitonidae, Melanopareiidae, Conopophagidae, Grallaridae, Rhinocryptidae, Scleruridae, Xenopidae, Pipridae, Cotingidae, Tityridae, Oxyruncidae, Onychorhynchidae, Pipritidae, Platyrinchidae, Tachurisidae, Rhynchocyclidae, Polioptilidae, Donacobiidae, Motacillidae, Fringillidae, Parulidae, and Mitrospingidae. The lack of known records of some taxa may be due to different taphonomic processes, paleontological fieldwork activities or simply because they were absent, as pointed out by Tambussi \& Noriega (1996), and these factors must be considered when analyzing fossil bird communities.

No families with no living representatives in the country but living elsewhere have records published. Of the globally extinct families, the following have fossil 
representatives in the country: Quercymegapodiidae (2), Pelagornithidae (dubious) (1), Palaelodidae (1), Teratornithidae (1), Gracilitarsidae (1), and Phorusrhacidae (3).

Sixteen new fossil genera and 21 species have been named, of seven extant and ten extinct families (equivalent clades): Cratoavis cearensis, Kaririavis mater, Diogenornis fragilis, Chaunoides antiquus, Neochen pugil, Taubacrex granivora, Ameripodius silvasantosi, Pelagornis longirostris, Agnopterus sicki, Hoazinavis lacustris, Ciconia lydekkeri, Anhinga minuta, Macranhinga ranzii, Brasilogyps faustoi, Wingegyps cartellei, Pleistovultur nevesi, Taubatornis campbelli, Eutreptodactylus itaboraiensis, Paleopsilopterus itaboraiensis, Itaboravis elaphrocnemoides, and Paraphysornis brasiliensis. The attribution of Pelagornis longirostris to Brazil is problematic. Additionally, two extinct species named from elsewhere have been found in the country, though one attribution is tentative: Rhea fossilis and Palaelodus cf. ambiguus.

Taxonomic uncertainty is present in 151 of the osteological records. However, even the positive records should not be taken without contestation due to the incomplete nature of the fossil record. Out of this number, 8 taxa have been determined to the subspecific level, all still living.

\subsection{Temporal representation}

Most of South America's Paleogene and Neogene birds have been described from the southern part of the continent (Argentina in particular), which also holds most of the Mesozoic remains found in the continent (Rasmussen \& Kay 1992; Agnolin 2016b). In Brazil, avian fossil material is known from the Early Cretaceous to the recent, holocenic record (extinct taxa are represented in temporal scale in Table 3), but there are still several temporal gaps to be filled.

Of the 378 taxa recorded, 74 are from Early Cretaceous, 7 from the Late Cretaceous, 14 from the Paleogene, 20 from the Paleogene-Neogene transition, 10 from the Neogene, and 253 from the Quaternary. 
Fossil feathers are most numerous in the late Aptian (70 records of indeterminate maniraptoran affinities), followed by late Oligocene-early Miocene (7 records), and Eocene-Oligocene (indeterminate number of specimens).

Eggs have one record in the Mesozoic (Campanian-Maastrichtian) and one in the Cenozoic (Oligocene-Miocene). Coprolites have a single record from the late Oligocene-early Miocene. A dubious track ichnofossil record is attributed to the late Cretaceous.

In sum, most Brazilian avian prehistoric material is known from the Cenozoic during the Pleistocene-Holocene transition.

\subsection{Spatial representation}

The political territory defined as Brazil currently has twenty-six states and the Federal District distributed into five Regions (North, Northeast, Central-West, Southeast, and South). Avian fossil remains are known from all Regions.

There are no published records from Amapá, Rondônia (North), Maranhão, Paraíba, Sergipe (Northeast), Mato Grosso do Sul, Federal District (Central-West), and Espírito Santo (Southeast).

The 378 taxa recorded here can be assigned to the following states: North: Acre (8), Amazonas (1), Pará (1), Roraima (1), Tocantins (5); Northeast: Alagoas (1), Bahia (12), Ceará (77), Pernambuco (4), Piauí (35), Rio Grande do Norte (4); Central-West: Goiás (13), Mato Grosso (1); Southeast: Minas Gerais (174), Rio de Janeiro (38), São Paulo (22); South: Paraná (1), Rio Grande do Sul (5), and Santa Catarina (1).

Early Cretaceous records are restricted to Ceará (Northeast) and Late Cretaceous to São Paulo and Minas Gerais (Southeast). Most of the Paleogene records come from Rio de Janeiro (Southeast), with meager records from Paraná (South) and Minas Gerais (Southeast). With exception of some late Miocene records of Acre and Amazonas (North), the late Cenozoic records are virtually restricted to cave remains of Quaternary age, which occur predominantly in Minas Gerais (Southeast) and Bahia and Piauí (Northeast). 
Two records of extinct species firstly described from elsewhere are present (Argentina and France), though one is uncertain.

\section{4. Preservation}

Following the four categories of preservation established in this study (i.e., feathers, eggs, ichnofossil, and osteological remains), the 378 recorded taxa can be divided into: 77 feathers, 2 eggs, 2 ichnofossils, and 297 osteological remains (including mummifications). Regarding the number of records in the literature for a taxon, 348 include a single record, 22 include two, seven include 3, and one include 4.

Regarding the degree of preservation of these specimens, we can classify them in low, medium, and high preservations. This is naturally somewhat arbitrary (since we were not able to examine every record in hands and the literature is not complete) and applies almost exclusively to osteological remains. This classification follows how much of the total specimen was preserved, low being just a few bones, medium including a reasonably good number of elements, and high staring from an almost complete skeleton. The 297 osteologically represented records can then be divided into: 220 low, 41 medium, 8 high, and 28 data deficient. Although rarely stated in material descriptions, most records in the literature belong to seemingly adult individuals. Feathers show a mostly reasonable preservation degree, with some specimens keeping their original coloration scheme. The oological records include one with good preservation and the other lacking sufficient information for a definition. Ichnofossils are reasonably well preserved.

\section{5. Final considerations}

As Bittencourt \& Langer (2012) pointed out concerning the few non-avian dinosaur species described for Brazil, the small number of fossil bird species known is also incongruent in the face of the country's continental dimensions and its rich sedimentary deposits, even considering the fragile nature of avian bones. Several actions could help increase the number of paleornithological studies in Brazil, including, as for scientific studies in general, better investments leading to new field work campaigns and creating a more stimulating scenario for new researchers, reanalyzing museum collections (in which avian bones, generally poor preserved, go unnoticed), enforcing laws on fossil 
traffic (which led valuable species into the scientific limbo of private collections), and geological sites preservation, and, as already pointed by Alvarenga (1992), expanding museum's osteological sections of ornithological collections.

Compiling and updating the knowledge on Brazilian bird remains gathered until the present is the first step for future studies to expand this knowledge, whether at a regional or national scale. Not only broadening the understanding of the taxonomic diversity and the evolution of birds, such expansion is important as a source of biogeographical and paleoecological information and may also help in future strategies for managing living species (Dietl \& Flessa 2011).

\section{REFERENCES}

Ackermann, F.L. (1964) Geologia e Fisiografia da Região Bragantina (Estado do Pará). Instituto Nacional de Pesquisas da Amazônia, Manaus, 90 pp.

Acosta Hospitaleche, C. \& Tambussi, C. (2005) Phorusrhacidae Psilopterinae (Aves) en la Formación Sarmiento de la localidad de Gran Hondonada (Eoceno Superior), Patagonia, Argentina. Revista Española de Paleontología, 20 (2), 127-132.

Agnolin, F. (2006a) Dos nuevos Anatidae (Aves, Anseriformes) del Pleistoceno inferior-medio de Argentina. Studia Geologica Salmanticensia, 42, 81-95.

Agnolin, F. (2006b) Presencia de Ciconia maltha (Aves, Ciconiidae) en el Pleistoceno Inferior-Medio del Valle de Tarija, Bolivia. Revista Española Paleontología, 21 (1), $39-41$.

Agnolin, F.L. (2007) Brontornis burmeisteri Moreno \& Mercerat, un Anseriformes (Aves) gigante del Mioceno Medio de Patagonia, Argentina. Revista del Museo Argentino de Ciencias Naturales (Nueva serie), 9 (1), 15-25.

Agnolin, F. (2009a) El registro fósil de Ciconia lydekkeri Ameghino, 1891 en el Pleistoceno de Sudamérica. Studia Geologica Salmanticensia, 45 (1), 53-58.

Agnolin, F. (2009b) Sistemática y Filogenia de las Aves Fororracoideas (Gruiformes, Cariamae). Fundación de Historia Natural Félix de Azara, Buenos Aires, 79 pp. 
Agnolin, F.L. (2013) La posición sistemática de Hermosiornis (Aves, Phororhacoidea) y sus implicancias filogenéticas. Revista del Museo Argentino de Ciencias Naturales (Nueva serie), 15 (1): 39-60.

Agnolin, F.L. (2016a) Unexpected diversity of ratites (Aves, Palaeognathae) in the early Cenozoic of South America: palaeobiogeographical implications. Alcheringa, 41 (1), $1-11$.

Agnolin, F.L. (2016b) A brief history of South American birds. Contribuciones del $M A C N, 6,157-172$.

Agnolin, F.L. \& Cenizo, M. (2014) Nuevos registros de aves fosíles argentinas y sus implicancias biogeográficas. XXVIII Jornadas Argentinas de Paleontología de Vertebrados (Resúmenes). Ameghiniana, 51 (6), Suplemento, 3.

Agnolin, F.L., Brissón Egli, F., Beilinson, E., Soibelzon, E., Rodriguez, S., Iacona, F. \& Soibelzon, L.H. (2016) New Cathartidae in the Quaternary of the Pampean Region (Argentina). Comments on the fossil record of condors. In: 9th International Meeting of the Society of Avian Paleontology and Evolution (Abstracts). SAPE, Diamante, pp. 2.

Agnolin, F.L., Brissón Egli, F., Soibelzon, E., Rodriguez, S.G., Soibelzon, L.H., Iacona, F. \& Piazza, D. (2017a) A new large Cathartidae from the quaternary of Argentina, with a review of the fossil record of condors in South America. Contribuciones del $M A C N, 7,1-16$.

Agnolin, F.L. \& Martinelli, A.G. (2009) Fossil birds from the Late Cretaceous Los Alamitos Formation, Río Negro Province, Argentina. Journal of South American Earth Sciences, 27 (1), 42-49.

Agnolin, F.L. \& Noriega, J.I. (2012) Una nueva especie de ñandú (Aves: Rheidae) del Mioceno tardío de la Mesopotamia argentina. Ameghiniana, 49 (2), 236-246.

Agnolin, F.L., Rozadilla, S. \& Carvalho, I.S. (2017b) Praeornis sharovi Rautian, 1978 a fossil feather from the early Late Jurassic of Kazakhstan. Historical Biology, 31 (7) (2019), 962-966. 
Aires, A.S.S. (2019) Notário em pterossauros e aves: aspectos evolutivos, ontogenéticos e morfo-funcionais. Pontifícia Universidade Católica do Rio Grande do Sul, Porto Alegre.

Aires, A.S., Reichert, L.M., Müller, R.T. \& Andrade, M.B. (2021) Review of morphology, development, and evolution of the notarium in birds. The Anatomical Record, 1-20, DOI: 10.1002/ar.24852.

Almeida, A.F., Anjos, D.A. \& Barbosa, L.F.W. (2005) Projeto de um robô animatrônico para simulação de movimentos de um animal pré-histórico. IX Encontro Latino Americano de Iniciação Científica e $V$ Encontro Latino Americano de PósGraduação - Universidade do Vale do Paraíba.

Almeida, R.C.V. (2003) Assimetria flutuante em esqueletos de aves Passeriformes do Estado de Minas Gerais: aspectos metodológicos e implicações sobre a utilização de coleções osteológicas como objeto de estudos de variação temporal de comunidades. Pontifícia Universidade Católica de Minas Gerais, Belo Horizonte, 57 pp.

Alt, L. \& Moura, V. (2020) Protection of the Environmental and Cultural Heritage in the Lagoa Santa Karst: History and Challenges. In: Auler, A.S. \& Pessoa, P. (Eds.) Lagoa Santa Karst: Brazil's Iconic Karst Region. Springer, Cham, pp. 305-330.

Alvarenga, H.M.F. (1982) Uma Gigantesca Ave Fóssil do Cenozóico Brasileiro: Physornis brasiliensis sp. n. Anais da Academia brasileira de Ciências, 54 (4), 697712.

Alvarenga, H.M.F. (1983) Uma ave ratitae do Paleoceno brasileiro: bacia calcária de Itaboraí, Estado do Rio de Janeiro, Brasil. Boletim do Museu Nacional, Nova Série, Geologia, 41, 1-8.

Alvarenga, H.M.F. (1985a) Um novo Psilopteridae (Aves: Gruiformes) dos sedimentos terciários de Itaboraí, Rio de Janeiro, Brasil. Anais do VIII Congresso Brasileiro de Paleontologia, Série Geologia 27, Paleontologia, Estratigrafia 2, 17-20. 
Alvarenga, H.M.F. (1985b) Notas sobre os Cathartidae (Aves) e Descrição de um Novo Gênero Cenozóico Brasileiro. Anais da Academia brasileira de Ciências, 57 (3), 349-357.

Alvarenga, H.M.F. (1988) Ave Fóssil (Gruiformes: Rallidae) dos Folhelhos da Bacia de Taubaté, Estado de São Paulo, Brasil. Anais da Academia brasileira de Ciências, 60 (3), 321-328.

Alvarenga, H.M.F. (1990) Flamingos Fósseis da Bacia de Taubaté, Estado de São Paulo, Brasil: Descrição de Nova Espécie. Anais da Academia brasileira de Ciências, 62 (4), 335-345.

Alvarenga, H.M.F. (1992) Coleções osteológicas: perspectivas para a ornitologia no Brasil. Boletim do Museu Paraense Emílio Goeldi, série Zoologia, 8 (1), 247-257.

Alvarenga, H. (1993a) Fossil Birds. In: Sick, H. Birds in Brazil: A Natural History. Princeton University Press, Princeton, pp. 62-64.

Alvarenga, H.M.F. (1993b) A origem das aves e seus fósseis. In: Andrade, M.A., A Vida das Aves: Introdução à Biologia e Conservação. Editora Líttera Maciel, Belo Horizonte, pp. 16-26.

Alvarenga, H.M.F. (1993c) Paraphysornis Novo Gênero Para Physornis brasiliensis Alvarenga, 1982 (Aves : Phorusrhacidae). Anais da Academia Brasileira de Ciências, 65 (4), 403-406.

Alvarenga, H.M.F. (1995a) A large and probably flightless Anhinga from the Miocene of Chile. Courier Forschungsinstitut Senckenberg, 181, 149-161.

Alvarenga, H.M.F. (1995b) Um Primitivo Membro da Ordem Galliformes (Aves) do Terciário Médio da Bacia de Taubaté, Estado de São Paulo, Brasil. Anais da Academia brasileira de Ciências, 67 (1), 33-44.

Alvarenga, H.F. (1997) Aves Fósseis: História da Origem e Evolução. In: Sick, H., Ornitologia Brasileira. Editora Nova Fronteira, Rio de Janeiro, pp. 120-123. 
Alvarenga, H.M.F. (1998) Sobre a Ocorrência do condor (Vultur gryphus) no Holoceno da Região de Lagoa Santa, Minas Gerais, Brasil. Ararajuba, 6 (1), 60-63.

Alvarenga, H.M.F. (1999) A Fossil Screamer (Anseriformes: Anhimidae) from the Middle Tertiary of Southeastern Brazil. Smithsonian Contributions to Paleobiology, $89,223-230$.

Alvarenga, H. (2003) Killer Birds: Ancient Predators of South America. South American Explorer, 72, 12-15.

Alvarenga, H. (2007a) O Museu de História Natural de Taubaté na Patagônia. Empresas do Vale, Ano 03, número 17.

Alvarenga, H. (2007) Anodorhynchus glaucus e A. leari (Psittaciformes, Psittacidae): osteologia, registros fósseis e antiga distribuição geográfica. Revista Brasileira de Ornitologia, 15 (3), 427-432.

Alvarenga, H. (2008) Um pedaço da história da Paraphysornis (...e do Museu também). Empresas do Vale, Ano 04, número 23.

Alvarenga, H. (2009) "Semana dos Museus" atesta avanço cultural na região. Empresas do Vale, Ano 05, número 29, 38.

Alvarenga, H. (2010) Diogenornis fragilis Alvarenga, 1985, restudied: a South American ratite closely related to Casuariidae. Abstracts of the $25^{\text {th }}$ International Ornithological Congress, 143.

Alvarenga, H.M.F. \& Bonaparte, J.F. (1992) A new flightless landbird from the Cretaceous of Patagonia. Natural History Museum of Los Angeles County, Science Series, 36, 51-64.

Alvarenga, H., Brito, G.R.R., Migotto, R., Hubbe, A. \& Höfling, E. (2008) Pleistovultur nevesi gen. et sp. nov. (Aves: Vulturidae) and the diversity of condors and vultures in the South American Pleistocene. Ameghiniana, 45 (3), 613-618. 
Alvarenga, H., Chiappe, L. \& Bertelli, S. (2011) Phorusrhacids: the Terror Birds. In: Dyke, G. \& Kaiser, G. (Eds.) Living Dinosaurs: The Evolutionary History of Modern Birds. Wiley-Blackwell, Chichester, pp. 187-208.

Alvarenga, H.M.F. \& Guilherme, E. (2003) The anhingas (Aves: Anhingidae) from the upper Tertiary (Miocene-Pliocene) of southwestern Amazonia. Journal of Vertebrate Paleontology, 23 (3), 614-621.

Alvarenga, H. \& Höfling, E. (2000) Aves. In: Carvalho, I.S. (Ed.) Paleontologia. Editora Interciência, Rio de Janeiro, pp. 579-593.

Alvarenga, H.M.F. \& Höfling, E. (2003) Systematic Revision of the Phorusrhacidae (Aves: Ralliformes). Papéis Avulsos de Zoologia, 43 (4), 55-91.

Alvarenga, H. \& Höfling, E. (2004) Aves. In: Carvalho, I.S. (Ed.) Paleontologia. $2^{a}$ Edição. Volume 1. Editora Interciência, Rio de Janeiro, pp. 817-831.

Alvarenga, H. \& Höfling, E. (2011) Aves. In: Carvalho, I.S. (Ed.) Paleontologia. $3^{a}$ Edição. Volume 3. Paleovertebrados, paleobotânica. Editora Interciência, Rio de Janeiro, pp. 113-133.

Alvarenga, H.M.F., Höfling, E. \& Silveira, L.F. (2002) Notharchus swainsoni (Gray, 1846) (Bucconidae) é uma espécie válida. Ararajuba, 10 (1), 73-77.

Alvarenga, H., Jones, W. \& Rinderknecht, A. (2010) The youngest record of phorusrhacid birds (Aves, Phorusrhacidae) from the late Pleistocene of Uruguay. Neues Jahrbuch für Geologie und Paläontologie, 256 (2), 229-234.

Alvarenga, H. \& Nava, W.R. (2005) Aves Enantiornithes do Cretáceo Superior da Formação Adamantina do Estado de São Paulo, Brasil. Boletim de Resumos do II Congresso Latino-Americano de Paleontologia de Vertebrados, 20.

Alvarenga, H.M.F. \& Olson, S.L. (2004) A new genus of tiny condor from the Pleistocene of Brazil (Aves: Vulturidae). Proceedings of the Biological Society of Washington, 117 (1), 1-9. 
Alvarenga, H., Ribeiro, G. \& Silva, D. (2007) Contribution of fossil birds to the age determination of the Tremembé Formation, Taubaté Basin, Brazil. Ameghiniana, 44 (4), Suplemento, 4R.

Alves, B.A. (2012a) Estudo do desgaste dentário de notoungulados (Mammalia) da Bacia de São José de Itaboraí, Rio de Janeiro (Itaboraiense). Universidade Federal do Rio de Janeiro, Rio de Janeiro, 81 pp.

Alves, T.R. (2012b) Análise filogenética de ralídeos Neotropicais (Aves: Rallidae) com base em caracteres osteológicos. Universidade de São Paulo, São Paulo, 84 pp.

Alves, Y.M., Bergqvist, L.P. \& Brito, P.M. (2016) New occurrences of microvertebrate fossil accumulations in Bauru Group, Late Cretaceous of western São Paulo state, Brazil. Journal of South American Earth Sciences, 69, 80-90.

Ameghino, F. (1882) Catálogo explicativo de las colecciones de antropología prehistórica y de paleontología de Florentino Ameghino. Catálogo de sección de la provincia de Buenos Aires, en la Exposición continental Sudamericana, Anexo A, $35-42$.

Ameghino, F. (1891) Enumeracion de las aves fósiles de la República Argentina. Revista Argentina de Historia Natural, 1, 441-453.

Amorim, C. (2006) Fóssil raro é exportado ilegalmente. O Estado de São Paulo, 17/07/2006, A15.

Andrade, J.A.F.G. (2007) Commercial exploitation of the Crato Formation. In: Martill, D.M., Bechly, G. \& Loveridge, R.F. (Eds.), The Crato Fossil Beds of Brazil: Window into an Ancient World. Cambridge University Press, Cambridge, pp. 63-69.

Andrade, R.O. (2017) O colecionador de ossos. Pesquisa FAPESP, 256, 98.

Andrela, S. \& Donatelli, R.J. (1995) Osteologia e miologia cranianas de Columbina squammata (Lesson, 1831) (Aves:Columbiformes). Naturalia, 20, 107-123.

Andrews, C.W. (1897). On Some Fossil Remains of Carinate Birds from Central Madagascar. The Ibis, 39 (3), 343-359. 
Angst, D., Buffetaut, E., Lecuyer, C. \& Amiot, R. (2016) A New Method for Estimating Locomotion Type in Large Ground Birds. Paleontology, 59 (2), 217-223.

Angst, D. \& Buffetaut, E. (2017) Paleobiology of Giant Flightless Birds. ISTE Press/Elsevier, London/ Kidlington, 296 pp.

Angst, D. \& Chinsamy-Turan, A. (2016) Ecological implications of the revised locomotion of the South American giant birds: Paraphysornis and Brontornis. 9th International Meeting of the Society of Avian Paleontology and Evolution (Abstracts), 3.

Antunes, A.Z., Alvarenga, H., Silveira, L.F., Eston, M.R., Menezes, G.V. \& Santos, A.S.R. (2007) Distribuição de Leptasthenura setaria (Temminck, 1824) (Aves: Furnariidae) no Estado de São Paulo. Biota Neotropical, 7 (1), 201-204.

Araújo, A.G.C.C. (2012) Diversidade morfológica dos ossos formadores do palatum osseum em Furnariides (Aves, Passeriformes, Tyranni). Universidade Federal do Estado do Rio de Janeiro, Rio de Janeiro.

Araújo, G.G. (2014) Osteologia comparada e análise filogenética da subfamília Picinae (Aves: Piciformes: Picidae). Universidade Federal do Estado do Rio de Janeiro, Rio de Janeiro.

Araújo Júnior, H.I. (2012) Tafonomia da acumulação fossilíferas de vertebrados pleistocênicos do Tanque do Jirau, Itapipoca, Estado do Ceará, Brasil. Universidade Federal do Rio de Janeiro, Rio de Janeiro, 185 pp.

Araújo Júnior, H.I. (2015) Modelo tafonômico para vertebrados de depósitos de tanque do Nordeste do Brasil. Universidade Federal do Rio de Janeiro, Rio de Janeiro, 193 pp.

Araújo Júnior, H.I. (2016) Classifying vertebrate assemblages preserved in Quaternary tank deposits: Implications for vertebrate taphonomy and paleoecology. Palaeogeography, Palaeoclimatology, Palaeoecology, 445, 147-152.

Araújo Júnior, H.I., Porpino, K.O., Ximenes, C.L. \& Bergqvist, L.P. (2013) Unveiling the taphonomy of elusive natural tank deposits: A study case in the Pleistocene of 
northeastern Brazil. Palaeogeography, Palaeoclimatology, Palaeoecology, 378, 5274.

Areta, J.I., Noriega, J.I. \& Agnolin, F. (2007) A giant darter (Pelecaniformes: Anhingidae) from the Upper Miocene of Argentina and weight calculation of fossil Anhingidae. Neues Jahrbuch für Geologie und Paläontologie, Abhandlungen, 243/3, $343-350$.

Auler, A.S. \& Piló, L.B. (2016) As descobertas de Lund. In: Da-Gloria, P., Neves, W.A. \& Hubbe, M. (Org.), Lagoa Santa: história das pesquisas arqueológicas e paleontológicas. Annablume Arqueológica, São Paulo, pp. 37-51.

Auler, A.S. (2020a) History of Research in the Lagoa Santa Karst. In: Auler, A.S. \& Pessoa, P. (Eds.) Lagoa Santa Karst: Brazil's Iconic Karst Region. Springer, Cham, pp. 1-11.

Auler, A.S. (2020b) Caves and Speleogenesis in the Lagoa Santa Karst. In: Auler, A.S. \& Pessoa, P. (Eds.) Lagoa Santa Karst: Brazil's Iconic Karst Region. Springer, Cham, pp. 167-186.

Averianov, A.O., Panteleyev, A.V., Potapova, O.R. \& Nessov, L.A. (1991) Bonytoothed Birds (Aves: Pelecaniformes: Odontopterygia) of the Late Paleocene and Eocene of the Western Margin of Ancient Asia. Trudy Zoologicheskogo Instituta, Akademiya Nauk SSSR, 239, 3-12.

Azevedo, R.P.F., Vasconcellos, P.L., Candeiro, C.R.A. \& Bergqvist, L.P. (2007) Restos microscópicos de vertebrados fósseis do Grupo Bauru (Neocretáceo), no Oeste do Estado de São Paulo, Brasil. In: Carvalho, I.S., Cassab, R.C.T., Schwanke, C., Carvalho, M.A., Fernandes, A.C.S., Rodrigues, M.A.C., Carvalho, M.S.S., Arai, M. \& Oliveira, M.E.Q. (Eds.) Paleontologia: Cenários de Vida. Volume 1. Editoria Interciência, Rio de Janeiro, pp. 541-549.

Azevedo, S.A. \& Carvalho, L.B. (1998) Avian eggshells from Tremembé Formation, Early Miocene of Brazil. Journal of Vertebrate Paleontology, 18 (3 suppl.), 25A. 
Baird, R.F. \& Vickers-Rich, P. (1997) Eutreptodactylus itaboraiensis gen. et sp. nov., an early cuckoo (Aves: Cuculidae) from the Late Paleocene of Brazil. Alcheringa, $21,123-127$.

Baird, R.F. \& Vickers-Rich, P. (1998) Palaelodus (Aves: Palaelodidae) from the Middle to Late Cainozoic of Australia. Alcheringa, 22, 135-151.

Bandeira, K.L.N., Brum, A.S., Souza, R.G. \& Campos, D.A. (2015) New material of anhinga (Suliformes, Anhuingidae) from Solimões Formation (Upper Miocene), Acre, Brazil. Paleo 2014, Súmula dos encontros e Resumos, Paleontologia em Destaque, 68, 115-116.

Baumel, J.J. \& Witmer, L.M. (1993) Osteologia. In: Baumel, J.J., King, A.S., Breazile J.E., Evans, H.E. \& Vanden Berge, J.C. (Eds.), Handbook of Avian Anatomy: Nomina Anatomica Avium. Second Edition. Publications of the Nuttall Ornithological Club, 23, pp. 45-132.

Becker, J.J. (1987) Additional material of Anhinga grandis Martin and Mengel (Aves: Anhingidae) from the Late Miocene of Florida. Proceedings of the Biological Society of Washington, 100 (2), 358-363.

Bedani, E.F. \& Haddad, C.F.B. (2002) Estudos preliminares de anfíbios (Anura:Pipidae) na Formação Entre-Córregos, Bacia de Aiuruoca, Terciário do Estado de Minas Gerais, Brasil. Geociências, 7 (6), 35-42.

Bergqvist, L.P., Mansur, K., Rodrigues, M.A., Rodrigues-Francisco, B.H., Perez, R. \& Beltrão, M.C. (2009) Bacia São José de Itaboraí, RJ - Berço dos mamíferos no Brasil. In: Winge, M., Schobbenhaus, C., Souza, C.R.G., Fernandes, A.C.S., BerbertBorn, M., Queiroz, E.T. \& Campos, D.A. (Eds.) Sítios Geológicos e Paleontológicos do Brasil - Volume II. CPRM, Brasília, pp. 413-432.

Bergqvist, L.P., Moreira, A.L. \& Pinto, D.R. (2006) Bacia de São José de Itaboraí: 75 Anos de História e Ciência. Serviço Geológico do Brasil - CPRM, Rio de Janeiro, 81 pp. 
Bernardes-de-Oliveira, M.E.C., Mandarim-de-Lacerda, A.F., Garcia, M.J. \& Campos, C.C. (2002) Fazenda Santa Fé, SP: A maior associação de fósseis do Terciário brasileiro. In: Schobbenhaus, C., Campos, D.A., Queiroz, E. T., Winge, M. \& Berbet-Born, M.L.C. (Eds.) Sítios Geológicos e Paleontológicos do Brasil. DNPM/CPRM - Comissão Brasileira de Sítios Geológicos e Paleobiológicos (SIGEP), Brasília, pp. 63-71.

Bertini, R.J., Marshall, L.G., Gayet, M. \& Brito, P. (1993) Vertebrate faunas from the Adamantina and Marília formations (Upper Baurú Group, late Cretaceous, Brazil) in their stratigraphic and paleobiogeographic context. Neues Jahrbuch für Geologie und Paläontologie, Abhandlungen, 188 (1), 71-101.

Bissaro-Júnior, M.C., Kerber, L., Crowley, J.L., Ribeiro, A.M., Ghilardi, R.P., Guilherme, E., Negri, F.R., Souza Filho, J.P. \& Hsiou, A.S. (2019) Detrital zircon $\mathrm{U}-\mathrm{Pb}$ geochronology constrains the age of Brazilian Neogene deposits from Western Amazonia. Palaeogeography, Palaeoclimatology, Palaeoecology, 516, 64-70.

Bittencourt, J.S. \& Langer, M.C. (2011) Mesozoic dinosaurs from Brazil and their biogeographic implications. Anais da Academia Brasileira de Ciências, 83 (1), 2360.

Bittencourt, J.S. \& Langer, M.C. (2012) Os dinossauros do Mesozoico brasileiro e as relações biogeográficas entre África e América do Sul. In: Gallo, V., Silva, H.M.A., Brito, P.M. \& Figueiredo, F.J. (Eds.) Paleontologia de Vertebrados: Relações entre América do Sul e África. Editoria Interciência, Rio de Janeiro, pp. 301-336.

Blanco, R.E. \& Jones, W.W. (2005) Terror birds on the run: a mechanical model to estimate its maximum running speed. Proceedings of the Royal Society B, 272, 1769-1773.

Bocquentin, J. \& Janoo, A. (1997) Uma nova espécie de Anhinga (Pelecaniformes, Anhingidae) proveniente do Neógeno da Amazônia sul-ocidental. 15 Congresso Brasileiro de Paleontologia (Boletim de Resumos), 111. 
Bocquentin, J. \& Melo, J. (2006) Stupendemys souzai sp. nov. (Pleurodira, Podocnemididae) from the Miocene-Pliocene of the Solimões Formation, Brazil. Revista Brasileira de Paleontologia, 9 (2), 187-192.

Bocquentin, J. \& Silva, E.G. (1998) Os Vertebrados Fósseis da Amazônia Sul-Ocidental do Brasil. Acta Geológica Lilloana, 18 (1), 149-197.

Boles, W.E. (2017) Fossil history of parrots. In: Forshaw, J.M. \& Knight, F., Vanished and Vanishing Parrots: Profiling Extinct and Endangered Species. CSIRO Publishing, Clayton South, pp. 1-12.

Borges, S.H. (2008) Bibliografia ornitológica do Brasil - dissertações e teses no período de 1970 à 2005. Revista Brasileira de Ornitologia, 16 (1), 82-98.

Bourdon, E., Ricqles, A. \& Cubo, J. (2009) A new Transantarctic relationship: morphological evidence for a Rheidae-Dromaiidae-Casuariidae clade (Aves, Palaeognathae, Ratitae). Zoological Journal of the Linnean Society, 156, 641-663.

Brito, G.R.R. (2008) Análise Filogenética de Cathartidae (Aves) com base em caracteres osteológicos. Universidade de São Paulo, São Paulo, 319 pp.

Brito, I.M. (1989) Geologia e paleontologia da bacia calcária de São José de Itaboraí, Estado do Rio de Janeiro, Brasil. Anuário do Instituto de Geociências, UFRJ, 12 , $56-64$.

Brodkorb, P. (1963) Catalogue of Fossil Birds: Part 1 (Archaeopterygiformes through Ardeiformes). Bulletin of the Florida State Museum, Biological Sciences, 7, 179293.

Brodkorb, P. (1964) Catalogue of Fossil Birds: Part 2 (Anseriformes through Galliformes). Bulletin of the Florida State Museum, Biological Sciences, 8 (3), 195335.

Brodkorb, P. (1965) Fossil birds from Barbados, West Indies. The Journal of the Barbados Museum and Historical Society, 31 (1), 3-10. 
Brodkorb, P. (1967) Catalogue of Fossil Birds: Part 3 (Ralliformes, Ichthyornithiformes, Charadriiformes). Bulletin of the Florida State Museum, Biological Sciences, 11 (3), 99-220.

Brodkorb, P. (1971) Catalogue of Fossil Birds: Part 4 (Columbiformes through Piciformes). Bulletin of the Florida State Museum, Biological Sciences, 15 (4), 163266.

Brodkorb, P. (1978) Catalogue of Fossil Birds: Part 5 (Passeriformes). Bulletin of the Florida State Museum, Biological Sciences, 23 (3), 139-228.

Brum, A.S. \& Simbras, F.M. (2015) The problematic Adamantina Formation (Santonian-Campanian), Bauru Group, Paraná Basin, and its theropod record. Paleo 2014, Súmula dos encontros e Resumos, Paleontologia em Destaque, 68, 119-120.

Bueno, L., Dias, A.S. \& Steele, J. (2013) The Late Pleistocene/Early Holocene archaeological record in Brazil: a geo-referenced database. Quaternary International, 301, 74-93.

Buffetaut, E. (2013) Who discovered the Phorusrhacidae? An episode in the history of avian paleontology. In: Göhlich, U.B. \& Kroh, A. (Eds.), Proceedings of the 8th International Meeting of the Society of Avian Paleontology and Evolution. Naturhistorisches Museum Wien, Wien, pp. 123-134.

Cabot, J., Jutglar, F., Garcia, E.F.J., Boesman, P., Sharpe, C.J. (2017a) Great Tinamou (Tinamus major). In: del Hoyo, J., Elliott, A., Sargatal, J., Christie, D.A. \& de Juana, E. (Eds.) Handbook of the Birds of the World Alive. Lynx Edicions, Barcelona. Retrieved from: http://www.hbw.com/node/52412 (accessed 20 July 2017).

Cabot, J., Jutglar, F., Garcia, E.F.J. \& Sharpe, C.J. (2017b) Solitary Tinamou (Tinamus solitarius). In: del Hoyo, J., Elliott, A., Sargatal, J., Christie, D.A. \& de Juana, E. (Eds.) Handbook of the Birds of the World Alive. Lynx Edicions, Barcelona. Retrieved from: http://www.hbw.com/node/52410 (accessed 20 July 2017). 
Caldas, S.S., Gomes, M.V.F., Silva, M.C.E.A., Fialho, F.S.F. \& Santana, M.I.S. (2019) Estudo descritivo e morfométrico do crânio do Ramphastos toco (Aves: Piciformes). Ciência Animal Brasileira, 20, e-44970, 1-14.

Calonge-Méndez, A. (1998) Osteologia craniana de Alcedinidae Neotropicais (Aves: Coraciiformes). Universidade de São Paulo, São Paulo, 104 pp.

Calonge-Méndez, A. (2004) Aspectos evolutivos e sistemáticos de Furnariidae (Aves: Passeriformes), com base em caracteres osteológicos. Universidade de São Paulo, São Paulo.

Calonge-Méndez, A. \& Höfling, E. (2007) Osteologia craniana de Cerylinae (Coraciiformes: Alcedinidae). Boletim do Museu Paraense Emílio Goeldi, Ciências Naturais, 2 (1), 155-182.

Camacho, I. \& Accorsi, M. (2016) Confirmação da sora, Porzana carolina, em território brasileiro e contribuições para a conservação das áreas úmidas da Área de Proteção Ambiental de Maricá (RJ) para espécies migratórias neárticas. Atualidades Ornitológicas, 191, 60-66.

Campbell Jr., K.E. (1992) A giant new Anhinga from the Miocene of Amazonia, with comments on gigantism in Miocene vertebrates of Amazonia. Fifty-second Annual Meeting, Society of Vertebrate Paleontology, Royal Ontario Museum (Abstracts of Papers). Journal of Vertebrate Paleontology, 12, supplement to number 3, 20A.

Campbell Jr., K.E. (1996) A New Species of Giant Anhinga (Aves: Pelecaniformes: Anhingidae) from the Upper Miocene (Huayquerian) of Amazonian Peru. Contributions in Science, 460, 1-9.

Campbell, M.O. (2016) Vultures: Their Evolution, Ecology and Conservation. CRC Press, Boca Raton, 374 pp.

Campos, A.P.C., Carvalho, R.T., Straker, L.C., Salgado, L.T., Kellner, A. \& Farina, M. (2019) Combined microscopy and spectroscopy techniques to characterize a fossilized feather with minimal damage to the specimen. Micron, 120, 17-24. 
Campos, H.B.N. (2011) Arcossauros da Bacia do Araripe: uma revisão. Tarairiú, 1 (2), 85-103.

Candeiro, C.R.A. (2005) Bauru group (late Cretaceous) vertebrates from Triângulo Minero region and western São Paulo state, Brazil: an introduction. Sociedade \& Natureza, 17 (32), 27-35.

Candeiro, C.R.A. (2015) Terópodes e Aves. In: Candeiro, C.R.A. \& Avilla, L.S. (Org.) Fósseis de Vertebrados e Plantas do Período dos Dinossauros da Região do Triângulo Mineiro. Letra Capital, Rio de Janeiro, pp. 160-169.

Candeiro, C.R.A., Agnolin, F., Martinelli, A.G. \& Buckup, P.A. (2012) First bird remains from the Upper Cretaceous of the Peirópolis site, Minas Gerais state, Brazil. Geodiversitas, 34 (3), 617-624.

Candeiro, C.R., Faria, C.C., Alves, M.C.M., Muniz, F., Alves, L., Vaz, M.T., Silva, R.M. \& Gil, L.M. (2013) Late Cretaceous tetrapods diversity of the Triângulo Mineiro Region (Minas Gerais State, Brazil). Revista Sapiência, 2 (2), 11-28.

Candeiro, C.R.A., Martinelli, A.G., Winter, C.P., Ribeiro, L.C.B. \& Bergqvist, L.P. (2010) Preliminary results of screenwashing in two Late Cretaceous outcrops of the Marilia Formation (Bauru Group) in the Triângulo Mineiro (Minas Gerais State, Brazil). $7^{o}$ Simpósio Brasileiro de Paleontologia de Vertebrados (Boletim de Resumos), 33 .

Cardia, F.M.S. (2019) Concentração de mercúrio em fósseis de vertebrados do Grupo Bauru: um novo método para reconstrução de cadeia trófica. XXVI Congresso Brasileiro de Paleontologia (Boletim de Resumos), Paleontologia em Destaque, Edição especial (Outubro/2019), 36.

Carleton, M.D. \& Olson, S.L. (1999) Amerigo Vespucci and the rat of Fernando de Noronha: a new genus and species of Rodentia (Muridae, Sigmodontinae) from a volcanic island off Brazil's continental shelf. American Museum Novitates, 3256, 159. 
Cartelle, C. (1983) Breve notícia dos achados paleontológicos na Gruta dos BrejõesBahia. X Congresso Brasileiro de Zoologia (Resumos), 442-444.

Cartelle, C. (1994) Tempo Passado, Mamíferos do Pleistoceno em Minas Gerais. Editora Palco, Belo Horizonte, 132 pp.

Cartelle, C. (2012) Das grutas à luz: os mamíferos pleistocênicos de Minas Gerais. Bicho do Mato Editora, Belo Horizonte, 236 pp.

Cartelle, C. (2016) Construindo o passado. Um olhar sobre a paleontologia de Lagoa Santa. In: Da-Gloria, P., Neves, W.A. \& Hubbe, M. (Org.), Lagoa Santa: história das pesquisas arqueológicas e paleontológicas. Annablume Arqueológica, São Paulo, pp. 323-346.

Cartelle, C. (2020) Cave Paleontology in the Lagoa Santa Karst. In: Auler, A.S. \& Pessoa, P. (Eds.) Lagoa Santa Karst: Brazil's Iconic Karst Region. Springer, Cham, pp. 209-225.

Cartelle, C., Abuhid, V., Ferreira, M.A.C. \& Ferreira, R.L. (1998) Levantamento Paleontológico. In: APA Carste de Lagoa Santa. Volume III. Patrimônio Espeleológico, Histórico e Cultural. IBAMA/CPRM, Belo Horizonte, pp. 1-6.

Cartelle, C. \& Santos, J.E. (1985) Notícia sobre a coleção osteológica de aves pleistocênicas da Gruta dos Brejões (BA) do Museu de Ciências Naturais da PUCMG. IX Congresso Brasileiro de Paleontologia (Resumos), 140.

Carvalho, I.S., Agnolin, F.L., Rozadilla, S., Novas, F.E., Ferreira Gomes Andrade, J.A. \& Xavier-Neto, J. (2021) A new ornithuromorph bird from the Lower Cretaceous of South America. Journal of Vertebrate Paleontology, DOI: 10.1080/02724634.2021.1988623.

Carvalho, I.S., Novas, F.E., Agnolín, F.L., Isasi, M.P., Freitas, F.I., Andrade, J.A. (2015a) A Mesozoic bird from Gondwana preserving feathers. Nature Communications, 6:7141, DOI: 10.1038/ncomms8141 [+Complementary material]. 
Carvalho I.S., Novas F.E., Agnolín F.L., Isasi M.P., Freitas F.I. \& Andrade, J.A. (2015b) A new genus and species of enantiornithine bird from the Early Cretaceous of Brazil. Brazilian Journal of Geology, 45 (2), 161-171.

Castilhos, W. (2005). Antigos vôos. Agência FAPESP. Available from: https://agencia.fapesp.br/antigos-voos/4162/ (accessed 04 November 2016).

Castro, A.C.J., Carvalho, I.S. \& Fernandes, A.C.S. (1988a) Introdução ao estudo dos coprólitos da Bacia de Taubaté. Anais da Academia Brasileia de Ciências, 60 (1), 109.

Castro, A.C.J., Fernandes, A.C.S. \& Carvalho, I.S. (1988b) Coprólitos de aves da Bacia de Taubaté, SP. Anais do XXXV Congresso Brasileiro de Geologia, 6, 2358-2370.

Castro-Fernandes, M.C., Bernardes-de-Oliveira, M.E.C. \& Hoelzel, A. (2013) Tafoflora Paleógena da Formação Entre-Córregos (Bacia de Aiuruoca): Arquitetura Foliar e Paleoclima. Geologia USP, Série científica, 13 (1), 35-46.

Cenizo, M.M. \& Agnolin, F. (2007) La presencia del género Belonopterus Reichenbach, 1852 (Aves, Charadriidae) en el Pleistoceno de Argentina, con la descripción de Belonopterus lilloi nov. sp. Revista del Museo Argentino de Ciencias Naturales, nueva serie, 9 (1), 41-47.

Cenizo, M.M. \& Agnolin, F.L. (2010) The southernmost records of Anhingidae and a new basal species of Anatidae (Aves) from the lower-middle Miocene of Patagonia, Argentina. Alcheringa, 34, 493-514.

Cenizo, M.M., Agnolin, F.L. \& Pomi, L.H. (2015) A New Pleistocene bird assemblage from the Southern Pampas (Buenos Aires, Argentina). Palaeogeography, Palaeoclimatology, Palaeoecology, 420, 65-81.

Chahud, A. (2001) Caracterização Tafonômica da Fauna de Vertebrados Fósseis (Pleistoceno-Holoceno) do Abismo Ponta de Flecha, Iporanga, SP. Universidade de São Paulo, São Paulo, 48 pp. 
Chávez, M. \& Stucchi, M. (2002) El registro de Pelagornithidae (Aves: Pelecaniformes) em el Pacifico sudeste. $1^{o}$ Congreso Latinoamericano de Paleontología de Vertebrados, 26.

Chávez, M., Stucchi, M. \& Urbina, M. (2007) El registro de Pelagornithidae (Aves: Pelecaniformes) y la avifauna neógena del Pacífico sudeste. Bulletin de l'Institut Français d'Études Andines, 36 (2), 175-197.

Chiappe, L.M. (1991) Cretaceous birds of Latin America. Cretaceous Research, 12, 5563.

Chiappe, L.M. (1996) Early Avian evolution in the Southern Hemisphere: The fossil record of birds in the Mesozoic of Gondwana. Memoirs of the Queensland Museum, 39 (3), 533-554.

Chiappe, L.M. (2007) Glorified Dinosaurs: The Origin and Early Evolution of Birds. John Wiley \& Sons, Hoboken, 263 pp.

Chiappe, L., Nava, W., Martinelli, A.G., Tucker, R., Abramowicz, S., Walsh, M. \& Alvarenga, H. (2019) An unique bonebed of enantiornithine birds from the Late Cretaceous of Southeast Brazil. XXVI Congresso Brasileiro de Paleontologia (Boletim de Resumos). Paleontologia em Destaque, Edição especial (Outubro/ 2019), 232-233.

Chiappe, L., Nava, W., Martinelli, A., Tucker, R. \& Alvarenga, H. (2018a) An exceptional bone bed of Enantiornithine birds in the late Cretaceous of Brazil. $5^{\text {th }}$ International Palaeontological Congress (Abstract Book), 170.

Chiappe, L.M., Nava, W., Martinelli, A.G., Tucker, R. \& Alvarenga, H. (2018b) A remarkable assemblage of Enantiornithine birds from the Late Cretaceous of Southeastern Brazil. $78^{\text {th }}$ Annual Meeting of the Society of Vertebrate Paleontology (Abstracts of Papers), 105.

Chim, E.N. (2018) Zooarqueologia da Lapa Grande de Taquaraçu. Museu de Arqueologia e Etnologia da Universidade de São Paulo, São Paulo, 260 pp. 
Cid, F.C. (2011) Filogenia dos Dendrocolaptidae com base em caracteres osteológicos. Universidade Federal do Estado do Rio de Janeiro, Rio de Janeiro.

Claussen, P. (1841) Notes géologiques sur la province de Minas Geraes au Brésil. Bulletin de l'Académie Royale des Sciences et Belles-Lettres de Bruxelles, 8 (5), $322-343$.

Close, R.A., Vickers-Rich, P., Trusler, P., Chiappe, L.M., O’Connor, J., Rich, T.H., Kool, L. \& Komarower, P. (2009) Earliest Gondwanan bird from the Cretaceous of Southeastern Australia. Journal of Vertebrate Paleontology, 29 (2), 616-619.

Collin, J. (1905) Winge, Gustav Oluf Bang. In: Bricka, C.F. (Ed.), Dansk Biografisk Lexikon. XIX Bind Vind-Oetken. Gyldendalske Boghandel, Nordisk Forlag, København, pp. 34.

Costa, T.V.V. (2014) Osteologia e filogenia das Aves Caprimulgiformes, com ênfase em Nyctibiidae e Caprimulgidae. Universidade de São Paulo, São Paulo, 454 pp.

Costa, T.V.V. \& Donatelli, R.J. (2009) Osteologia craniana de Nyctibiidae (Aves, Caprimulgiformes). Papéis Avulsos de Zoologia, 49 (21), 257-275.

Costa, T.V.V., Silveira, L.F., Posso, S.R. \& Donatelli, R.J. (2021) Phylogenetic analysis of the nocturnal avian family Nyctibiidae (Caprimulgiformes) inferred from osteological characters. Zoologischer Anzeiger, 291, 113-122.

Costa, T.V.V., Whitney, B.M., Braun, M.J., White, N.D., Silveira, L.F. \& Cleere, N. (2017) A systematic reappraisal of the Rufous Potoo Nyctibius bracteatus (Nyctibiidae) and description of a new genus. Journal of Ornithology, 159, 367-377.

Couto-Ribeiro, G. (2010) Avaliação morfológica, taxonômica e cronológica dos mamíferos fósseis da Formação Tremembé (Bacia de Taubaté), Estado de São Paulo, Brasil. Universidade de São Paulo, São Paulo, 99 pp.

Cozzuol, M.A. (2006) The Acre vertebrate fauna: Age, diversity, and geography. Journal of South American Earth Sciences, 21, 185-203. 
Cuello, J.P. (1988) Lista de las aves fósiles de la región neotropical y de las islas antillanas. Paula-Coutiana, 2, 3-79.

Cunha, R.F. (2016) Contexto paleoambiental e tafonomia da assembléia fóssil da Formação Guabirotuba, Bacia de Curitiba, Paraná. Universidade Federal do Paraná, Curitiba, 120 pp.

Cunha, R.F., Dias, E., Sedor, F.A. \& Fernandes, L.A. (2014) Taphonomic aspects of vertebrate fossils on the Guabirotuba Formation (Curitiba Basin, Paleogene), Paraná, Brazil. Abstract Volume - 4th International Palaeontological Congress, 691.

Davis, P.G. \& Briggs, D.E.G. (1995) Fossilization of feathers. Geology, 23 (9), 783786.

Dénes, F.V. \& Silveira, L.F. (2007) Cranial osteology and taxonomy of albatrosses of genus Diomedea Linnaeus, 1758 and Thalassarche Reichenbach, 1853 (Procellariiformes: Diomedeidae). Papéis Avulsos de Zoologia, 47 (3), 43-61.

Dias, A.S. (2004) Diversificar para poblar: El contexto arqueológico brasileño en la transición Pleistoceno-Holoceno. Complutum, 15, 249-263.

Dias-Brito, D., Musacchio, E.A., Castro, J.C., Maranhão, M.S.A.S., Suarez, J.M. \& Rodrigues, R. (2001) Grupo Bauru: uma unidade continental do Cretáceo no Brasil concepções baseadas em dados micropaleontológicos, isotópicos e estratigráficos. Revue de Paleobiologie, 20 (1), 245-304.

Diederle, J.M. (2015) Los Anhingidae (Aves: Suliformes) del Neógeno de América del Sur: sistemática, filogenia y paleobiología. Universidad Nacional de La Plata, La Plata, 327 pp.

Diederle, J.M. (2016) Body mass and locomotor habits of the smallest darter, Anhinga minuta (Aves, Anhingidae). Historical Biology, 29 (3), 289-295.

Diederle, J.M. (2017) Taxonomic status of the Neogene snakebird Anhinga fraileyi (Aves, Anhingidae). Ameghiniana, 54 (3), 341-347. 
Diederle, J.M. \& Agnolin, F. (2017) New anhingid (Aves, Suliformes) from the middle Miocene of Río Negro province, Patagonia, Argentina. Historical Biology, 29 (8), 1056-1064.

Dietl, G.P. \& Flessa, K.W. (2011) Conservation paleobiology: putting the dead to work. Trends in Ecology \& Evolution, 26 (1), 30-37.

Donatelli, R.J. (1992) Cranial osteology and myology of the jaw apparatus in the Galbulidae (Aves, Piciformes). Arquivos de Zoologia, 32 (1) 1-32.

Donatelli, R.J. (1996) The jaw apparatus of the Neotropical and of the Afrotropical woodpeckers (Aves: Piciformes). Arquivos de Zoologia, 33 (1), 1-70.

Donatelli, R.J. (1997) Osteologia e miologia cranianas de Dendrocolaptidae (Passeriformes, Tyranni) 1. Gêneros Glyphorhynchus, Campylorhamphus, Dendrocincla, Xiphorhynchus e Dendrocolaptes. Ararajuba, 5 (1), 19-37.

Donatelli, R.J. (2012a) Cranial osteology of the Picini (Aves: Piciformes: Picidae). Acta Zoologica, doi: 10.1111/azo.12014, 1-11.

Donatelli, R.J. (2012b) Cranial Osteology of Meiglyptini (Aves: Piciformes: Picidae). Anatomy Research International, 2012, 951836, 1-9.

Donatelli, R.J. \& Marceliano, M.L.V. (2007) Osteologia e miologia cranianas de Megaxenops parnaguae (Furnariidae: Philydorinae). Boletim do Museu Paraense Emílio Goeldi, Ciências Naturais, 2 (1), 183-215.

Dyke, G.J. (2003) Cenozoic Birds of the World, Part I: Europe, by Jirí Mlíkovsky, 2002 (Review). Journal of Vertebrate Paleontology, 23 (1), 258.

Elliott, A., Boesman, P. \& Kirwan, G.M. (2015) Maguari Stork (Ciconia maguari). In: del Hoyo, J., Elliott, A., Sargatal, J., Christie, D.A. \& de Juana, E. (Eds.) Handbook of the Birds of the World Alive. Lynx Edicions, Barcelona. Retrieved from: http://www.hbw.com/node/52743 (accessed 30 December 2015).

Elliott, A., Garcia, E.F.J., Kirwan, G.M. \& Boesman, P. (2015) Jabiru (Jabiru mycteria). In: del Hoyo, J., Elliott, A., Sargatal, J., Christie, D.A. \& de Juana, E. 
(Eds.) Handbook of the Birds of the World Alive. Lynx Edicions, Barcelona. Retrieved from: http://www.hbw.com/node/52748 (accessed 30 December 2015).

Emslie, S.D. (1988) The fossil history and phylogenetic relationships of condors (Ciconiiformes: Vulturidae) in the New World. Journal of Vertebrate Paleontology, $8(2), 212-228$.

Faure, M., Guérin, C. \& Mourer-Chauviré, C. (2010) L'Art rupestre du Parc National Serra da Capivara (Piauí, Brésil): Bestiaire figuré et données paleontologiques. Anais do Congresso Internacional de Arte Rupestre IFRAO (2009). FUMDHAMentos, IX (2), 1-15.

Feduccia, J.A. (1967) Ciconia maltha and Grus canadensis from the Upper Pliocene of Idaho. Wilson Bulletin, 79, 316-318.

Fernandes, L.A. (2004) Mapa litoestratigráfico da parte oriental da Bacia Bauru (PR, SP, MG), escala 1:1.000.000. Boletim Paranaense de Geociências, 55, 53-66.

Fernandes, L.A. \& Coimbra, A.M. (1996) A Bacia Bauru (Cretáceo Superior, Brasil). Anais da Academia Brasileira de Ciências, 68 (2), 195-205.

Fernandes, L.A. \& Coimbra, A.M. (2000) Revisão estratigráfica da parte oriental da Bacia Bauru (Neocretáceo). Revista Brasileira de Geociências, 30, 717-728.

Ferraroni, A. (2015) Taxonomia e filogenia de Aratinga Spix, 1824 (Aves: Psittacidae). Universidade de São Paulo, São Paulo, 389 pp.

Ferreira, C.D. (2007) Filogenia dos Ciconiiformes (Aves) com ênfase em Threskiornithidae. Universidade Estadual Paulista, Botucatu, 184 pp.

Ferreira, C.D. \& Donatelli, R.J. (2005) Osteologia craniana de Platalea ajaja (Linnaeus) (Aves, Ciconiiformes), comparada com outras espécies de Threskiornithidae. Revista Brasileira de Zoologia, 22 (3), 529-551.

Ferreira, R.L., Souza, M., Santana, R. \& Silva, M.S. (2005) Bioespeleologia e Paleontologia. In: IBAMA. Plano de Manejo Parque Nacional Cavernas do Peruaçu, Encarte 3: Análise da UC, 150-161. 
Figuti, L. (2005) A Arqueofauna do Sítio de Santa Elina: Nota Preliminar. In: Vialou, A.V. (Ed.), Pré-história do Mato Grosso. Volume 1: Santa Elina. Edusp, São Paulo, pp. 155-158.

Fisher, H.I. (1944) The skulls of the cathartid vultures. The Condor, 46 (6), 272-296.

Flausino Jr., O.A., Donatelli, R.J. \& Pascotto, M.C. (1999) Osteologia e miologia cranianas de Momotus momota (Coraciiformes, Momotidae). Ararajuba, 7 (2), 109124.

Fonseca, S. (2019a) Pesquisadores encontram novos fósseis de aves, crocodilo e peixes em sítio paleontológico em Presidente Prudente. G1. Available from: https://g1.globo.com/sp/presidente-prudenteregiao/noticia/2019/05/16/pesquisadores-encontram-novos-fosseis-de-avescrocodilo-e-peixes-em-sitio-paleontologico-em-presidente-prudente.ghtml (accessed 17 May 2019).

Fonseca, S. (2019b) Descoberta de fósseis raros de aves da 'Era dos Dinossauros' em Presidente Prudente completa 15 anos. G1. Available from: https://g1.globo.com/sp/presidente-prudente-regiao/noticia/2019/09/26/descobertade-fosseis-raros-de-aves-da-era-dos-dinossauros-em-presidente-prudente-completa15-anos.ghtml (accessed 26 September 2019).

Foth, C. (2020) A Morphological Review of the Enigmatic Elongated Tail Feathers of Stem Birds. In: Foth, C. \& Rauhut, O.W.M. (Eds.), The Evolution of Feathers: From Their Origin to the Present. Springer, Cham, pp. 173-184.

Frailey, C.D. (1986) Late Miocene and Holocene mammals, exclusive of the Notoungulata, of the Acre region, western Amazonia. Contributions in Science, 374, $1-43$.

Francisco, B.H.R.; Souza Cunha, F.L. (1978) Geologia e Estratigrafia da Bacia de São José, Município de Itaboraí, RJ. Anais da Academia Brasileira de Ciências, 50 (3), $381-416$ 
Franco-Delgado, S.G. \& Bernardes-de-Oliveira, M.E.C. (2004) Annonaceae e Lauraceae da Formação Entre-Córregos (Paleógeno) na Bacia de Aiuruoca: implicações paleoclimáticas. Revista Brasileira de Paleontologia, 7 (2): 117-126.

Freitas, W.G.S., Silva, J.S., Silva, W.M., Eleutério, L.H.S. \& Sayão, J.M. (2010) Criação da coleção paleontológica do Centro Acadêmico de Vitória (UFPE-CAV). Paleo 2010 Nordeste (Resumos), 32.

Gaban-Lima, R. (2007) Análise Filogenética de Psittaciformes (Aves) com base em caracteres morfológicos siringeais e osteológicos. Universidade de São Paulo, São Paulo, 203 pp.

Galvão, P. \& Peñaranda, J. (2020) The Geology of Lagoa Santa Karst. In: Auler, A.S. \& Pessoa, P. (Eds.) Lagoa Santa Karst: Brazil’s Iconic Karst Region. Springer, Cham, pp. 13-25.

Garcia, M.J. (1993) Coleção do projeto "A Paleontologia da Formação Tremembé", Terciário do Estado de São Paulo. Anais da Academia Brasileira de Ciências, 65 (3), 329.

Garcia, M.J. \& Saad, A.R. (1996) Estado-da-arte do Projeto: “A Paleontologia da Formação Tremembé (Bacia de Taubaté), Oligoceno do Estado de São Paulo". Revista UnG, 2, 36-45.

Garcia, M.J., Santos, M. \& Hasui, Y. (2000) Palinologia da parte aflorante da Formação Entre-Córregos, Bacia de Aiuruoca, Terciário do Estado de Minas Gerais, Brasil. Revista Universidade Guarulhos, Geociências, 5, 259.

Gervais, P. (1844a) M. Paul Gervais communique à la Société le résumé suivant d'un travail qu'il a entrepris et qui a pour titre: Remarques sur les Oiseaux fossiles. L'Institut, XII (557), 293-294.

Gervais, P. (1844b) Remarques sur les oiseaux fossiles. Thèse de Géologie. Imprimerie de Poussielgue, Paris, 40 pp.

Goedert, J.L. (1989) Giant Late Eocene Marine Birds (Pelecaniformes: Pelagornithidae) from Northwestern Oregon. Journal of Paleontology, 63 (6), 939-944. 
Goeldi, E.A. (1894) As Aves do Brasil. Primeira Parte. Livraria Classica de Alves \& C, Rio De Janeiro.

Góes, A.M., Rosseti, D.F., Nogueira, A.C.R. \& Toledo, P.M. (1990) Modelo deposicional preliminar da Formação Pirabas no nordeste do estado do Pará. Boletim do Museu Paraense Emílio Goeldi, Série Ciências da Terra, 2, 3-15.

Greco, M.C. \& Cozzuol, M.A. (2012) O acervo de Paleontologia do Museu de História Natural e Jardim Botânico da UFMG. Arquivos do Museu de História Natural e Jardim Botânico, 21 (1), 149-154.

Grellet-Tinner, G., Murelaga, X., Larrasoaña, J.C., Silveira, L.F., Olivares, M., Ortega, L.A., Trimby, P.W. \& Pascual, A. (2012) The First Occurrence in the Fossil Record of an Aquatic Avian Twig-Nest with Phoenicopteriformes Eggs: Evolutionary Implications. PLoS ONE, 7 (10), e46972.

Guérin, C. (1993) La faune pléistocène de la Lagoa da Pedra à Conceição das Creoulas / Salgueiro, Pernambouc, Brésil. CLIO - Série Arqueológica, 9, 15-20.

Guerin, C., Faure, M.., Simões, P.R., Hugueney, M. \& Mourer-Chauvire, C. (2002) Toca da Janela da Barra do Antonião, São Raimundo Nonato, PI - Rica fauna pleistocênica e registro da Pré-história brasileira. In: Schobbenhaus, C., Campos, D.A., Queiroz, E.T., Winge, M. \& Berbert-Born, M.L.C (Eds.) Sítios Geológicos e Paleontológicos do Brasil. DNPM/CPRM/SIGEP, Brasília, pp. 131-137.

Guérin, C., Hugueney, M., Mourer-Chauviré, C. \& Faure, M. (1993a) Paléoenvironnement pléistocène dans l'aire archéologique de São Raimundo Nonato (Piaui, Brésil) : apport des mammifères et des oiseaux. Documents des laboratoires de géologie de la Faculté des sciences de Lyon, 125, 187-202.

Guérin, C., Curvello, M.A., Faure, M., Hugueney, M. \& Mourer-Chauviré, C. (1993b) La faune pleistocene du Piaui (Nordeste du Bresil): implications paleoecologiques et biochronologiques. Quaternaria Nova, 3, 303-341. 
Guérin, C., Curvello, M.A., Faure, M., Hugueney, M. \& Mourer-Chauviré, C. (1996) A fauna pleistocênica do Piauí (Nordeste do Brasil): relações paleoecológicas e biocronológicas. FUMDHAMentos, I (1), 55-103.

Guidon, N. (1991) Peintures préhistoriques du Brésil. L'art rupestre du Piauí. Editions Recherches sur les Civilisations, Paris, 109 pp.

Guidon, N., Pessis, A.M. \& Martin, G. (2009a) Pesquisas Arqueológicas na Região do Parque Nacional Serra da Capivara e seu entorno (Piauí - 1998 - 2008). FUMDHAMentos, VIII, 2-61.

Guidon, N., Guérin, C., Faure, M., Felice, G.D., Buco, C. \& Ignácio, E. (2009b) Toca das Moendas, Piauí-Brasil, primeiros resultados das escavações arqueológicas. FUMDHAMentos, VIII, 71-85.

Guilherme, E. (2016) Aves do Acre. Edufac, Rio Branco, 897 pp.

Guilherme, E., Souza, L.G.D., Loboda, T.S., Ranzi, A., Adamy, A., Dos Santos Ferreira, J. \& Souza-Filho, J.P. (2020) New material of Anhingidae (Aves: Suliformes) from the upper Miocene of the Amazon, Brazil. Historical Biology, 33 (11) [2021], 3091-3100.

Guzzi, A. (2007) Filogenia dos Ciconiidae (Aves, Ciconiiformes) com base em caracteres osteológicos cranianos. Universidade Estadual Paulista, Botucatu, 257 pp.

Guzzi, A., Branco, M.S.D. \& Donatelli, R.J. (2016) Cranial osteology of the genus Sclerurus (Passeriformes: Furnariidae). Revista de Biología Tropical, 64 (30), 11551170.

Guzzi, A., Castro, J.M.N., Martins Junior, R.N.C., Donatelli, R.J., Soares, L.M.S. \& Ferreira, G.J.B.C. (2015a) Osteologia craniana comparada de Caracara cheriway (Jacquin, 1784) e Caracara plancus (Miller, 1777) (Aves: Falconidae). Ciência e Natura, 37 (2), 218-229. 
Guzzi, A., Nascimento, M.S., Lima, S.P., Santos, S.S. \& Donatelli, R.J. (2014a) Osteologia craniana e aspectos evolutivos de Mycteria (Aves: Ciconiidae). Revista Nordestina de Biologia, 23 (1), 85-102.

Guzzi, A., Santos, F.C.V., Ferreira, G.J.B.C. \& Donatelli, R.J. (2014b) Comparative analysis of the cranial osteology and evolutionary traits of Micrastur gilvicollis, Micrastur ruficollis and Micrastur semitorquatus (Aves: Falconiformes). Revista Nordestina de Zoologia, 8 (1), 70-88.

Guzzi, A., Santos, A.M., Santos, J.D., Donatelli, R.J. \& Ferreira, G.J.B.C. (2015b) Principais caracteres da osteologia craniana de Milvago chimango (Vieillot, 1816) e Milvago chimachima (Vieillot, 1816) (Aves: Falconidae). Biotemas, 28 (3), 107-119.

Hansen, K.L. (2012) E Museo Lundii - Addendum. Statens Naturhistoriske Museum, København, 103 pp.

Harrison, C.J.O. (1975) A re-examination of the extinct Pleistocene Stork Palaeociconia australis. Bulletin of the British Ornithologists' Club, 95 (3), 94-96.

Harrison, C.J.O. \& Walker, C.A. (1976) A review of the bony-toothed birds (Odontopterygiformes): with descriptions of some new species. Tertiary Research Special Paper, 2, 1-62.

Harrison, C.J.O. (1985) A bony-toothed Bird (Odontopterygiformes) from the Palaeocene of England. Tertiary Research, 7 (1), 23-25.

Heimhofer, U. \& Martill, D.M. (2007) The sedimentology and depositional environment of the Crato Formation. In: Martill, D.M., Bechly, G. \& Loveridge, R.F. (Eds.), The Crato Fossil Beds of Brazil: Window into an Ancient World. Cambridge University Press, Cambridge, pp. 44-62.

Hellmayr, C.R. (1906) Revision der Spix'schen Typen brasilianischer Vögel. Abhandlungen der Mathematisch-Physikalischen Klasse der Königlich Bayerischen Akademie der Wissenschaften, 22 (3), 561-726. 
Hellmayr, C.E. \& Conover, B. (1942) Catalogue of Birds of the Americas and the Adjacent Islands in Field Museum of Natural History, Part 1, Number 1. Zoological Series, 13.

Helmreichen, V. (1846) Rio de Janeiro, am 6. Mai 1846. In: Hocheder, J.K. (1847), Virgil von Helmreichens Reise in Brasilien. Berichte über die Mittheilungen von Freunden der Naturwissenschaften in Wien, 2 (10), pp. 138-151.

Helms, O. (1932) Kort Udsigt over Dansk Ornithologi fra de ældste Tider til omkring aar 1900. Dansk Ornitologisk Forenings Tidsskrift, 26 (3), 81-102.

Herzog, A., Sales, A. \& Hillmer, G. (2008) The UNESCO Araripe Geopark: A short story of the evolution of life, rocks and continents. Expressão Gráfica e Editora, Fortaleza, $71 \mathrm{pp}$.

Höfling, E. (1991) Etude comparative du crâne chez des Ramphastidae (Aves, Piciformes). Bonner zoologische Beiträge, 42 (1), 55-65.

Höfling, E. (1995) Anatomia do crânio e da cintura escapular dos Ramphastidae (Aves: Piciformes) e de alguns grupos próximos, com implicações sistemáticas. Universidade de São Paulo, São Paulo, 185 pp.

Höfling, E. (2004) Recent anatomical studies on Neotropical birds. Ornitología Neotropical, 15 (Suppl.), 73-81.

Höfling, E. \& Alvarenga, H.M.F. (2001) Osteology of the Shoulder Girdle in the Piciformes, Passeriformes and Related Groups of Birds. Zoologischer Anzeiger, 240, 196-208.

Höfling, E. \& Gasc, J.P. (1984a) Biomécanique du crâne et du bec chez Ramphastos (Ramphastidae, Aves), I. Description des éléments anatomiques. Gegenbaurs morphologisches Jahrbuch, 130 (1), 125-147.

Höfling, E. \& Gasc, J.P. (1984b) Biomécanique du crâne et du bec chez Ramphastos (Ramphastidae, Aves), II. Analyse des conditions du mouvement du bec. Gegenbaurs morphologisches Jahrbuch, 130 (2), 235-262. 
Holt, D.W., Berkley, R., Deppe, C., Enríquez Rocha, P., Petersen, J.L., Rangel Salazar, J.L., Segars, K.P., Wood, K.L. \& de Juana, E. (2017) Tawny-browed Owl (Pulsatrix koeniswaldiana). In: del Hoyo, J., Elliott, A., Sargatal, J., Christie, D.A. \& de Juana, E. (Eds.) Handbook of the Birds of the World Alive. Lynx Edicions, Barcelona. Retrieved from: http://www.hbw.com/node/55054 (accessed 9 July 2017).

Holten, B. \& Sterll, M. (2011) Peter Lund e as grutas com ossos em Lagoa Santa. Editora UFMG, Belo Horizonte, 335 pp.

Hopson, J.A. (1964) Pseudodontornis and other large marine birds from the Miocene of South Carolina. Postilla, 83, 1-19.

Howard, H. (1964) Fossil Anseriformes. In: Delacour, J., The Waterfowl of the World. Volume 4. Country Life Limited, London, pp. 233-326.

Howard, H. \& Warter, S.L. (1969) A new species of bony-toothed bird (Family Pseudodontornithidae) from the Tertiary of New Zealand. Records of the Canterbury Museum, 8 (4), 345-357.

Hubbe, A. (2008) Contextualização taxonômica, tafonômica e morfométrica dos remanescentes ósseos da megamastofauna da Gruta Cuvieri (MG), um sítio paleontológico do Pleistoceno Tardio. Universidade de São Paulo, São Paulo, 141 pp.

Hubbe, A., Haddad-Martim, P.M., Hubbe, M., Mayer, E.L., Strauss, A., Auler, A.S., Piló, L.B. \& Neves, W.A. (2011) Identification and importance of critical depositional gaps in pitfall cave environments: The fossiliferous deposit of Cuvieri Cave, eastern Brazil. Palaeogeography, Palaeoclimatology, Palaeoecology, 312, 6678.

HRH The Prince Philip, Duke of Edinburgh \& Fisher, J. (1970) Wildlife Crisis. Cowles Book Company, New York, 256 pp.

Hsiou, A.S. (2007) O estado atual do registro fóssil de répteis e aves no Pleistoceno do Estado do Rio Grande do Sul, Brasil. In: Bauermann, S.G., Ribeiro, A.M., 
Rodrigues, P.H., Scherer, C.S., Evaldt, A.P.C. \& Corrêa, M.V.G. (Org.) Quaternário do Rio Grande do Sul: Integrando Conhecimentos (Resumos), pp. 20-22.

Hsiou, A.S. (2009a) O registro fóssil de répteis e aves no Pleistoceno final do Estado do Rio Grande do Sul, Brasil. In: Ribeiro, A.M., Bauermann, S.G. \& Scherer, C.S. (Org.). Quaternário do Rio Grande do Sul: Integrando Conhecimentos. Monografias da Sociedade Brasileira de Paleontologia. Pallotti, Porto Alegre, pp 143-154.

Hsiou, A.S. (2009b) Répteis e Aves do Pleistoceno do Estado do Rio Grande do Sul. In: Da-Rosa, A.A.S. (Org.) Vertebrados Fósseis de Santa Maria e Região. Pallotti, Santa Maria, pp. 321-338.

Hume, J.P. (2017) Extinct Birds. Second Edition. Bloomsbury, London, 608 pp.

Hurt, W.R. \& Blasi, O. (1969) O Projeto Arqueológico "Lagoa Santa" - Minas Gerais, Brasil (Nota final). Arquivos do Museu Paranaense, Nova Série, Arqueologia, 4, 163.

International Commission on Zoological Nomenclature (2000) International Code of Zoological Nomenclature. Fourth edition. Available from: http://www.iczn.org (accessed 13 March 2017).

Jacobus, A.L., Gazzaneo, M. \& Momberger, S. (1988) Presença de pinguim-demagalhães, Spheniscus magellanicus (Sphenisciformes, Spheniscidae), em sítios arqueológicos do Sul do Brasil. Resumos do XV Congresso Brasileiro de Zoologia, 465.

Jones, W.W. (2010) Nuevos aportes sobre la paleobiología de los fororrácidos (Aves: Phorusrhacidae) basados en el análisis de estructuras biológicas. Universidad de la República, Facultad de Ciencias - PEDECIBA, Montevideo, 213 pp.

Kachniasz, K.E. \& Silva-Caminha, S.A.F. (2013) Composição palinológica de Patos e Niterói, no Acre: inferências sobre idade e paleoambiente. Boletim de Resumos do XXIII Congresso Brasileiro de Paleontologia, Paleontologia em Destaque, Edição Especial (Outubro/2013), 128. 
Kellner, A.W.A. (1998) Panorama e Perspectiva do Estudo de Répteis Fósseis no Brasil. Anais da Academia Brasileira de Ciências, 70 (3), 647-676.

Kellner, A.W.A. (2002) A Review of Avian Mesozoic Fossil feathers. In: Chiappe, L.M. \& Witmer, L.M. (Eds.) Mesozoic Birds: Above the Heads of Dinosaurs. University of California Press, Berkeley, pp. 389-404.

Kellner, A. (2006) Pterossauros: os senhores do céu do Brasil. Vieira \& Lent, Rio de Janeiro, $176 \mathrm{pp}$.

Kellner, A.W.A. \& Campos, D.A. (1999) Vertebrate paleontology in Brazil - a review. Episodes, 22 (3), 238-251.

Kellner, A.W.A. \& Campos, D.A. (2000) Brief Review of Dinosaur Studies and Perspectives in Brazil. Anais da Academia Brasileira de Ciências, 72 (4), 509-538.

Kellner, A.W.A., Maisey, J.G. \& Campos, D.A. (1994) Fossil down feather from the Lower Cretaceous of Brazil. Palaeontology, 37 (3), 489-492.

Kellner, A.W.A., Martins-Neto, R.G. \& Maisey, J.G. (1991) Undetermined feather. In: Maisey, J.G. (Ed.) (1991) Santana Fossils: An Illustrated Atlas. T.F.H. Publications, Neptune City, pp. 376-377.

Kellner, A.W.A., Schwanke, C. \& Campos, D.A. (1999) O Brasil no tempo dos dinossauros. Museu Nacional, Rio de Janeiro, 60 pp.

Kerber, L. \& Oliveira, E.V. (2008a) Fósseis de vertebrados da Formação Touro Passo (Pleistoceno Superior), Rio Grande do Sul, Brasil: atualização dos dados e novas contribuições. Journal of Geoscience, 4 (2), 49-64.

Kerber, L. \& Oliveira, E.V. (2008b) Novos Fósseis de Vertebrados para a Sanga da Cruz (Pleistoceno Superior), Alegrete, RS, Brasil. Revista Pesquisas em Geociências, 35 (2), 39-45.

Kerber, L., Pitana, V.G., Ribeiro, A.M., Hsiou, A.S. \& Oliveira, E.V. (2014) Late Pleistocene vertebrates from Touro Passo Creek (Touro Passo Formation), southern Brazil: a review. Revista Mexicana de Ciencias Geológicas, 31(2), 248-259. 
Kipnis, R., Bissaro Jr., M.C. \& Prado, H.M. (2010a) Os restos faunísticos. In: Araujo, A.G.M. \& Neves, W.A. (Orgs.), Lapa das Boleiras: Um Sítio Paleoíndio do Carste de Lagoa Santa, MG, Brasil. Annablume, São Paulo, pp. 121-147.

Kipnis, R., Santos, R.O. \& Cesário, M.E. (2010b) A indústria óssea. In: Araujo, A.G.M. \& Neves, W.A. (Orgs.), Lapa das Boleiras: Um Sítio Paleoíndio do Carste de Lagoa Santa, MG, Brasil. Annablume, São Paulo, pp. 111-119.

Klein, V.C. \& Ferreira, C.S. (1979) Paleontologia e Estratigrafia de uma Facies Estuarina da Formação Itapecuru, Estado do Maranhão. Anais da Academia Brasileira de Ciências, 51 (3), 523-533.

Kneip, L.M. (1977) Pescadores e coletores pré-históricos do litoral de Cabo Frio, RJ. Revista do Museu Paulista, Série de Arqueologia, 5, 7-139.

Kneip, L.M. (2001) O Projeto Saquarema e as pesquisas realizadas. Documento de Trabalho, 5, 1-13.

Kneip, L.M., Coelho, A.C.S., Souza Cunha, F.L. \& Mello, E.M.B. (1975) Informações preliminares sobre a arqueologia e a fauna do Sambaqui do Forte, Cabo Frio, RJ. Revista do Museu Paulista, Nova Série, 22, 89-108.

Kneip, L.M. (Coord.), Crancio, F., Pallestrini, L., Mello, E.M.B., Corrêa, M.M.G, Magalhães, R.M.M., Vogel, M.A.C, Campinha, C.M., Moraes Junior, D.F., Verissimo, S.G. \& Barros, F.N. (1994) Cultura material e subsistência das populações pré-históricas de Saquarema, RJ. Documento de trabalho, 2, 1-120.

Kneip, L.M., Araújo, D.S.D. \& Fonseca, V.S. (1995) Áreas de exploração de recursos abióticos e bióticos das populações pré-históricas de Saquarema, RJ. Documento de Trabalho, 3, 3-11.

Kneip, L.M., Crancio, F., Santos, C.M.C., Magalhães, R.M.M. \& Mello, E.M.B. (1997) O Sambaqui do Saco e de Madressilva - Saquarema, RJ. Documento de Trabalho, 4, $1-67$.

Knoll, F., Chiappe, L.M., Sanchez, S., Garwood, R.J., Edwards, N.P., Wogelius, R.A., Sellers, W.I., Manning, P.L., Ortega, F., Serrano, F.J., Marugán-Lobón, J., Cuesta, 
E., Escaso, F. \& Sanz, J.L. (2018) A diminutive perinate European Enantiornithes reveals an asynchronous ossification pattern in early birds. Nature Communications, 9: 937, DOI: 10.1038/s41467-018-03295-9.

Krabbe, N. (2007) Birds collected by P. W. Lund and J. T. Reinhardt in south-eastern Brazil between 1825 and 1855, with notes on P. W. Lund's travels in Rio de Janeiro. Revista Brasileira de Ornitologia, 15 (3), 331-357.

Ksepka, D.T. (2014) Flight performance of the largest volant bird. Proceedings of the National Academy of Sciences of the United States of America, 111 (29), 1062410629.

Ksepka, D.T. \& Habib, M. (2016) Giants of the Sky. Scientific American, 314, 64-71.

Ladeira, L.M.C.E.B. \& Höfling, E. (2007) Osteologia craniana de Bucconidae. Boletim do Museu Paraense Emílio Goeldi, Ciências Naturais, 2 (1), 117-153.

Lambrecht, K. (1930) Studien über fossile Riesenvögel. Geologica Hungarica, Series Palaeontologica, 7, 1-37.

Lambrecht, K. (1933) Handbuch der Palaeornithologie. Gebrüder Borntraeger, Berlin, 1024 pp.

Laming-Emperaire, A., Prous, A., Vilhena de Moraes, A., Beltrão, M. \& Leme, J.L. (1975) Grottes et abris de la région de Lagoa Santa, Minas Gerais, Brésil. Cahiers d'archéologie d'Amérique du Sud, 1, 1-185.

Langer, M.C., Montefeltro, F.C., Castro, M.C., Marsola, J.C.A., França, M.A.G., Vila Nova, B., Kerber, L. \& Rabello, F. (2013) Fósseis de Caiman da Gruta Ioiô, Bahia, Brasil. Boletim de Resumos do XXIII Congresso Brasileiro de Paleontologia, Paleontologia em Destaque, Edição Especial (Outubro/2013), 239.

Latrubesse, E.M., Bocquentin, J., Santos, J.C.R. \& Ramonell, C.G. (1997) Paleoenvironmental model for the late Cenozoic of Southwestern Amazonia: paleontology and geology. Acta Amazonica, 27 (2), 103-118. 
Latrubesse, E.M., Cozzuol, M., Silva-Caminha, S.A.F., Rigsby, C.A., Absy, M.L. \& Jaramillo, C. (2010) The Late Miocene paleogeography of the Amazon Basin and the evolution of the Amazon River system. Earth-Science Reviews, 99, 99-124.

Latrubesse, E.M.; Silva, S.A.F.; Cozzuol, M.; Absy, M.L. (2007) Late Miocene continental sedimentation in southwestern Amazonia and its regional significance: Biotic and geological evidence. Journal of South American Earth Sciences, 23, 6180 .

Leite K.J.G. \& Hessel, M.H. (2011) Novas evidências de pequenos dinossauros no Membro Crato da Formação Santana, Aptiano da Bacia do Araripe, Brasil. Anais do XI Encontro de Pós-Graduação e Pesquisa da Universidade de Fortaleza, 1, 1-5.

Leonhard, K.C. \& Bronn, H.G. (Eds.) (1843) Neues Jahrbuch für Mineralogie, Geognosie, Geologie und Petrefakten-Kunde. E. Schweizerbart'sche Verlagshandlung, Stuttgart, 868 pp.

Lessa, G., Souza, F.H. \& Boroni, N.L. (2020) The Lagoa Santa Fauna: Historical Records. In: Auler, A.S. \& Pessoa, P. (Eds.) Lagoa Santa Karst: Brazil's Iconic Karst Region. Springer, Cham, pp. 51-72.

Liais, E. (1872) Climats, géologie, faune et géographie botanique du Brésil. Garnier Frères, Paris, 640 pp.

Lima, M.R. (1989) Fósseis do Brasil. EDUSP, São Paulo, 118 pp.

Lima, U.D.B., Azevedo, S.A.K. \& Grillo, O.N. (2008) Reconstrução e Digitalização de Paraphysornis brasiliensis Empregando Modelagem Virtual Tridimensional. Universidade Federal do Rio de Janeiro, Rio de Janeiro.

Livezey, B.C. (1997) A Phylogenetic Classification of Waterfowl (Aves: Anseriformes), including Selected Fossil Species. Annals of Carnegie Museum, 66 (4), 457-496.

Loboda, T.S., Souza, L.G., Guilherme, E., Toledo, P.M., Ranzi, A., Adamy, A., Ferreira, J.S. \& Souza-Filho, J.P. (2019) Cajueiro: A remarkable site of Solimoes Formation (Upper Miocene) discovered at Purus river by "Expedição Profa. Rosalie 
Benchimol. XXVI Congresso Brasileiro de Paleontologia (Boletim de Resumos), 136.

Lönnberg, E. (1902) On some fossil remains of a Condor from Bolivia. Bulletin of the Geological Institution of the University of Upsala, 6 (1), 1-11.

Lopes, A.M., Souza, V. \& Holanda, E.C. (2011) Levantamento paleontológico nas Formações Serra do Tucano, Tacutu e Manari, Bacia do Tacutu - RR. $12^{\circ}$ Simpósio de Geologia da Amazônia, 1-4.

Lopes, R.J. (2005) Ave brasileira conviveu com dinossauros. Folha de S.Paulo, 11/ago/2005, A22.

Lopes, R.J. (2020) Cidade paulista deve se tornar principal jazida de fósseis de aves da Era dos Dinossauros no país. Folha de S.Paulo. Available from: https://www1.folha.uol.com.br/ciencia/2020/10/cidade-paulista-deve-se-tornarprincipal-jazida-de-fosseis-de-aves-da-era-dos-dinossauros-no-pais.shtml (accessed 20 March 2021).

Lopes, R.P., Brião, C. \& Buchmann, F.S.C. (2006a) Primeiro registro de fósseis pleistocênicos de aves marinhas na planície costeira do Estado do Rio Grande do Sul, Brasil. Boletim da Sociedade Brasileira de Paleontologia, 53, 45.

Lopes, R.P., Buchmann, F.S.C. \& Caron, F. (2006b) Primeiro registro de fósseis de aves marinhas na planície costeira do Rio Grande do Sul, Brasil. Revista Brasileira de Geociência, 36 (4), 648-650.

Lopes, R.P., Pereira, J.C. \& Ferigolo, J. (2019) A Late Pleistocene fossil stork (Ciconiiformes: Ciconiidae) from the Santa Vitória Formation, Southern Brazil and its paleoenvironmental significance. Revista Brasileira de Paleontologia, 22 (3), $199-216$.

Lund, P.W. (1837) Om Huler i Kalksteen i det indre af Brasilien, der tildeels indeholde fossile knokler, Förste Afhandling. Det Kongelige Danske Videnskabernes Selskabs Naturvidenskabelige og Mathematiske Afhandlinger, 6, 207-248. 
Lund, P.W. (1839) Coup-d'oeil sur les espèces éteintes de Mammifères du Brésil; extrait de quelques mémoires présentés à l'Académie royale des Sciences de Copenhague. Annales des Sciences Naturelles, Seconde Série, Zoologie, 11, 214234.

Lund, P.W. (1840) Nouvelles Recherches sur la Faune fossile du Brésil. Annales des Sciences Naturelles, Seconde Série, Zoologie, 13, 310-319.

Lund, P.W. (1841a) Blik paa Brasiliens Dyreverden för Sidste Jordomvæltning, Förste Afhandling: Indledning. Det Kongelige Danske Videnskabernes Selskabs Naturvidenskabelige og Mathematiske Afhandlinger, 8, 27-60.

Lund, P.W. (1841b) Blik paa Brasiliens Dyreverden för Sidste Jordomvæltning, Anden Afhandling: Pattedyrene. Det Kongelige Danske Videnskabernes Selskabs Naturvidenskabelige og Mathematiske Afhandlinger, 8, 61-144.

Lund, P.W. (1841c) Blik paa Brasiliens Dyreverden för Sidste Jordomvæltning, Tredie Afhandling: Fortsættelse af Pattedyrene. Det Kongelige Danske Videnskabernes Selskabs Naturvidenskabelige og Mathematiske Afhandlinger, 8, 217-272.

Lund, P.W. (1841d) Lagoa Santa den 30. Januar 1841. Oversigt over det Kongelige danske Videnskabernes Selskabs Forhandlinger og dets Medlemmers Arbeider, $1841,16-18$.

Lund, P.W. (1842a) Lagoa Santa den 30te Januar 1841. Det Kongelige Danske Videnskabernes Selskabs Naturvidenskabelige og Mathematiske Afhandlinger, 9, LXI-LXIII.

Lund, P.W. (1842b) Fortsatte Bemærkninger over Brasiliens Uddöde Dyrskabning. Det Kongelige Danske Videnskabernes Selskabs Naturvidenskabelige og Mathematiske Afhandlinger, 9, 121-136.

Lund, P.W. (1842c) Blik paa Brasiliens Dyreverden för Sidste Jordomvæltning, Fjerde Afhandling: Fortsættelse af Pattedyrene. Det Kongelige Danske Videnskabernes Selskabs Naturvidenskabelige og Mathematiske Afhandlinger, 9, 137-208. 
Lund, P.W. (1843) Mittheilungen an Professor Bronn gerichtet. Lagoa Santa in Brasilien, 6. Juni 1843. In: Leonhard, K.C. \& Bronn, H.G. (Eds.), Neues Jahrbuch für Mineralogie, Geognosie, Geologie und Petrefakten-Kunde. E. Schweizerbart'sche Verlagshandlung, Stuttgart, pp. 785-786.

Lund, P.W. (1845-1849) Notice sur des Ossements Humains Fossiles, Trouvés dans une Caverne du Brésil. Mémoires de la Société Royale des Antiquaires du Nord, 1845-1849: 49-77.

Lund, P.W. (1846) Meddelelse af Det Udbytte de i 1844 undersögte knoglehuler Have afgivet til kundskaben om Brasiliens Dyreverden för Sidste Jordomvæltning. Det Kongelige Danske Videnskabernes Selskabs Naturvidenskabelige og Mathematiske Afhandlinger, 12, 57-94.

Lydekker, R. (1891) Catalogue of the fossil birds in the British Museum (Natural History). Taylor and Francis, London, 368 pp.

Machado, E.B. \& Kellner, A.W.A. (2007) On a supposed ornithischian dinosaur from the Santana Formation, Araripe Basin, Brazil. In: Carvalho, I.S., Cassab, R.C.T., Schwanke, C., Carvalho, M.A., Fernandes, A.C.S., Rodrigues, M.A.C., Carvalho, M.S.S., Arai, M. \& Oliveira, M.E.Q. (Eds.) Paleontologia: Cenários de Vida. Volume 1. Editoria Interciência, Rio de Janeiro, pp. 299-307.

Magalhães, R.M.M., Curvelo, M.A., Mello, E.M.B. (2001) A Fauna na Alimentação. Documento de Trabalho, 5, 55-63.

Mahecha, G.A.B. \& Oliveira, C.A. (1998) An Additional Bone in the Sclera of the Eyes of Owls and the Common Potoo (Nictibius griseus) and Its Role in the Contraction of the Nictitating Membrane. Acta Anatomica, 163, 201-211.

Mallet-Rodrigues, F. (2016) The ornithology of the state of Rio de Janeiro in postgraduate courses in Brazil. Atualidades Ornitológicas, 192, 57-65.

Marantz, C., Aleixo, A., Bevier, L. \& Patten, M. (2003) Family Dendrocolaptidae (Woodcreepers). In: del Hoyo, J.; Elliot, A.; Christie, D. (Eds.) (2003) Handbook of 
the Birds of the World. Volume 8: Broadbills to Tapaculos. Lynx Edicions, Barcelona, pp. 358-447.

Marceliano, M.L.V. (1996). Estudo osteológico e miológico do crânio de Opisthocomus hoazin (Müller, 1776) (Aves: Opisthocomidae), comparado com algumas espécies de Cracidae, Musophagidae e Cuculidae. Boletim do Museu Paraense Emílio Goeldi, série Zoologia, 12 (2) 95-246.

Marceliano, M.L.V., Donatelli, R.J., Höfling, E. \& Posso, S.R. (1997) Osteologia e miologia cranianas de Psophiidae (Aves: Gruiformes). Boletim do Museu Paraense Emílio Goeldi, série Zoologia, 13 (1), 39-76.

Marceliano, M.L.V., Donatelli, R.J. \& Posso, S.R. (2007a) Osteologia e miologia cranianas de Eurypyga helias (Pallas, 1781) (Gruiformes: Eurypygidae) comparada com outros Gruiformes. Boletim do Museu Paraense Emílio Goeldi, Ciências Naturais, 2 (1), 69-86.

Marceliano, M.L.V., Donatelli, R.J. \& Posso, S.R. (2007b) Osteologia craniana de Geotrygon montana (Linnaeus, 1758) (Columbiformes: Columbidae) comparada com os Columbiformes do Novo Mundo. Boletim do Museu Paraense Emílio Goeldi, Ciências Naturais, 2 (3), 21-31.

Marceliano, M.L.V., Posso, S.R. \& Donatelli, R.J. (2007c) Osteologia craniana de Penelope superciliaris Temminck, 1815 (Galliformes: Cracidae). Boletim do Museu Paraense Emílio Goeldi, Ciências Naturais, 2 (1), 55-67.

Marsola, J.C.A. (2013) Novos registros de ovos fósseis de vertebrados do Cretáceo Superior do Grupo Bauru. Universidade de São Paulo, Ribeirão Preto, 81 pp.

Marsola, J.C.A., Grellet-Tinner, G., Montefeltro, F.C., Sayão, J.M., Hsiou, A.S. \& Langer, M.C. (2014) The first fossil avian egg from Brazil. Alcheringa, 38 (4), 563567.

Marsola, J.C.A., Hsiou, A.S., Montefeltro, F.C., Langer, M.C., Grellet-Tinner, G., Sayão, J.M. \& Ghilardi, R.P. (2012) Ocorrência de um ovo de Aves no Cretáceo 
Superior do Grupo Bauru. VIII Simpósio Brasileiro de Paleontologia de Vertebrados (Boletim de Resumos), 49.

Martill, D.M. (1993) Fossils of the Santana and Crato Formations, Brazil. The Palaeontological Association, London, 159 pp.

Martill, D.M. (1994) Fake fossils from Brazil. Geology Today, 10 (3), 111-115.

Martill, D.M. (2007) The geology of the Crato Formation. In: Martill, D.M., Bechly, G. \& Loveridge, R.F. (Eds.), The Crato Fossil Beds of Brazil: Window into an Ancient World. Cambridge University Press, Cambridge, pp. 8-24.

Martill, D.M. \& Bechly, G. (2007) Introduction to the Crato Formation. In: Martill, D.M., Bechly, G. \& Loveridge, R.F. (Eds.), The Crato Fossil Beds of Brazil: Window into an Ancient World. Cambridge University Press, Cambridge, pp. 3-7.

Martill, D.M., Bechly, G. \& Loveridge, R.F. (Eds.) (2007) The Crato Fossil Beds of Brazil: Window into an Ancient World. Cambridge University Press, Cambridge, $625 \mathrm{pp}$.

Martill, D.M. \& Davis, P.G. (1998) Did dinosaurs come up to scratch? Nature, 396, $528-529$.

Martill, D.M. \& Davis, P.G. (2001) A feather with possible ectoparasite eggs from the Crato Formation (Lower Cretaceous, Aptian) of Brazil. Neues Jahrbuch für Geologie und Paläontologie, Abhandlungen, 219 (3), 241-259.

Martill, D.M. \& Filgueira, J.B.M. (1994) A new feather from the Lower Cretaceous of Brazil. Palaeontology, 37 (3), 483-487.

Martill, D.M. \& Frey, E. (1995) Colour patterning preserved in Lower Cretaceous birds and insects: The Crato Formation of N.E. Brazil. Neues Jahrbuch für Geologie und Paläontologie, Monatshefte, 1995 (2), 118-128.

Martill, D.M. \& Heimhofer, U. (2007) Stratigraphy of the Crato Formation. In: Martill, D.M., Bechly, G. \& Loveridge, R.F. (Eds.), The Crato Fossil Beds of Brazil: Window into an Ancient World. Cambridge University Press, Cambridge, pp. 25-43. 
Martin, G. (1996) Pré-história do Nordeste do Brasil. Universitária da UFPE, Recife, $395 \mathrm{pp}$.

Martin, L.D. \& Mengel, R.M. (1975) A new species of anhinga (Anhingidae) from the Upper Pliocene of Nebraska. The Auk, 92, 137-140.

Martinelli, A.G. \& Teixeira, V.P.A. (2015) The Late Cretaceous vertebrate record from the Bauru Group in the Triângulo Mineiro, southeastern Brazil. Boletín Geológico y Minero, 126 (1), 129-158.

Martins-Neto, R.G. \& Kellner, A.W.A. (1988) Primeiro Registro de Pena na Formação Santana (Cretáceo Inferior), Bacia do Araripe, Nordeste do Brasil. Anais da Academia Brasileira de Ciências, 60 (1), 61-68.

Martins Neto, R.G. \& Pesenti, M. (2006) The First Fossil Termitidae (Isoptera) from the Oligocene of South America: the Entre-Córregos Formation of the Aiuruoca Basin, Minas Gerais, Brazil. Journal of the Entomological Research Society, 8 (3), 63-68.

Matheu, E. del Hoyo, J., Bonan, A., Garcia, E.F.J. \& Boesman, P. (2017a). Plumbeous Ibis (Theristicus caerulescens). In: del Hoyo, J., Elliott, A., Sargatal, J., Christie, D.A. \& de Juana, E. (Eds.) Handbook of the Birds of the World Alive. Lynx Edicions, Barcelona. Retrieved from: http://www.hbw.com/node/52767 (Accessed 25 July 2017).

Matheu, E., del Hoyo, J., Garcia, E.F.J. \& Boesman, P. (2017b). Sharp-tailed Ibis (Cercibis oxycerca). In: del Hoyo, J., Elliott, A., Sargatal, J., Christie, D.A. \& de Juana, E. (Eds.) Handbook of the Birds of the World Alive. Lynx Edicions, Barcelona. Retrieved from: http://www.hbw.com/node/52770 (Accessed 25 July 2017).

Matheu, E., del Hoyo, J., Garcia, E.F.J., Kirwan, G.M. \& Boesman, P. (2017c). Blackfaced Ibis (Theristicus melanopis). In: del Hoyo, J., Elliott, A., Sargatal, J., Christie, D.A. \& de Juana, E. (Eds.) Handbook of the Birds of the World Alive. Lynx Edicions, Barcelona. Retrieved from: http://www.hbw.com/node/52769 (Accessed 25 July 2017). 
Mayer, E.L. (2011) Processos de formação de um depósito fossilífero em abismo na Gruta Cuvieri (MG): taxonomia, tafonomia e distribuição espacial de fósseis de mamíferos do Pleistoceno. Universidade de São Paulo, São Paulo, 145 pp.

Mayr, E. \& Amadon, D. (1951) A Classification of Recent Birds. American Museum Novitates, 1496, 1-42.

Mayr, G. (2001) A New Specimen of the Tiny Middle Eocene Bird Gracilitarsus mirabilis (New Family: Gracilitarsidae). The Condor, 103 (1), 78-84.

Mayr, G. (2005) Phylogenetic affinities and composition of the early Eocene Gracilitarsidae (Aves, ?Piciformes). Neues Jahrbuch für Geologie und Paläontologie, Monatshefte, 2005 (1), 1-16.

Mayr, G. (2008) A skull of the giant bony-toothed bird Dasornis (Aves: Pelagornithidae) from the Lower Eocene of the Isle of Sheppey. Palaeontology, 51 (5), 1107-1116.

Mayr, G. (2009) Paleogene Fossil Birds. Springer, Heidelberg, 280 pp.

Mayr, G. (2014) A hoatzin fossil from the middle Miocene of Kenya documents the past occurrence of modern-type Opisthocomiformes in Africa. The Auk, 131, 55-60.

Mayr, G. (2016) Avian Evolution: The Fossil Record of Birds and Its Paleobiological Significance. Wiley-Blackwell, West Sussex, 320 pp.

Mayr, G., Alvarenga, H. \& Clarke, J.A. (2011a) An Elaphrocnemus-like landbird and other avian remains from the late Paleocene of Brazil. Acta Palaeontologica Polonica, 56 (4), 679-684.

Mayr, G., Alvarenga, H. \& Mourer-Chauviré, C. (2011b) Out of Africa: Fossils shed light on the origin of the hoatzin, an iconic Neotropic bird. Naturwissenschaften, 98, 961-966.

Mayr, G. \& De Pietri, V.L. (2014) Earliest and first Northern Hemispheric hoatzin fossils substantiate Old World origin of a "Neotropic endemic". Naturwissenschaften, 101, 143-148. 
Mayr, G., Goedert, J.L. \& McLeod, S.A. (2013) Partial skeleton of a bony-toothed bird from the Late Oligocene/Early Miocene of Oregon (USA) and the systematics of Neogene Pelagornithidae. Journal of Paleontology, 87 (5), 922-929.

Mayr, G. \& Rubilar-Rogers, D. (2010) Osteology of a New Giant Bony-toothed Bird from the Miocene of Chile, with a Revision of the Taxonomy of Neogene Pelagornithidae. Journal of Vertebrate Paleontology, 30 (5), 1313-1330.

McEvey, A. (1968) Books, Papers and Literary Notes. The Emu, 68 (3), 223-225.

McKee, J.W.A. (1985) A pseudodontorn (Pelecaniformes: Pelagornithidae) from the middle Pliocene of Hawera, Taranaki, New Zealand. New Zealand Journal of Zoology, 12, 181-184.

Melo, D.J. \& Bergqvist, L.P. (2010) A paleobiodiversidade de vertebrados e inferências paleoecológicas da Formação Tremembé, Bacia de Taubaté/SP (Deseadense). $7^{o}$ Simpósio Brasileiro de Paleontologia de Vertebrados (Boletim de Resumos): 94.

Melo, D.J., Bergqvist, L.P. \& Alvarenga, H.M.F. (2007) Considerações Paleoecológicas sobre os Notoungulados da Formação Tremembé, Bacia de Taubaté (São Paulo, Brasil). Anuário do Instituto de Geociências - UFRJ, 30 (1), 77-82.

Mercerat, A. (1897) Note sur les oiseaux fossiles de la République Argentine. Anales de la Sociedad Científica Argentina, 43, 222-240.

Metello, T. (2013) Diogenornis fragilis, the oldest ratite: claws, locomotor behavior and taxonomic implications. $73^{\text {rd }}$ Annual Meeting of Society of Vertebrate Paleontology (Abstracts of Papers): 176.

Metello, T.M. (2017) Diversidade de penas fósseis da Bacia do Araripe (Formação Santana, Membro Crato), Aptiano. Universidade Federal do Rio de Janeiro, Rio de Janeiro, 44 pp.

Metello, T.M. \& Araújo Júnior, H.I. (2013) Um Gavião Fóssil no Pleistoceno do Ceará. Paleo 2012: Súmula dos Encontros e Resumo (Paleontologia em Destaque 66), 6970. 
Metello, T.M. \& Bergqvist, L.P. (2014) Claws of flightless birds from Itaboraí basin (Paleocene): locomotor behavior and palebiology. $4^{\text {th }}$ International Palaeontological Congress (Abstract Volume), 151.

Metello, T.M., Lopes, L.N.F., Melki, L.B., Bergqvist, L.P. \& Marinho, T.S. (2012a) Uma Nova Falange de Ave Ratita da Bacia de São José de Itaboraí (Paleoceno Superior) do Estado do Rio de Janeiro. XXXIV Jornada Giulio Massarani de Iniciação Científica, Artística e Cultural UFRJ (Livro de resumos), 525.

Metello, T.M., Pereira, P.V.L.G.C. \& Bergqvist, L.P. (2014) Garras fósseis da Bacia de São José de Itaboraí (Paleoceno Superior). IX Simpósio Brasileiro de Paleontologia de Vertebrados (Boletim de Resumos), 84.

Metello, T.M., Taranto, R.C., Pereira, P.V.L.G.C. \& Bergqvist, L.P. (2012b) Primeira descrição de garra aviana da Bacia São José de Itaboraí, Rio de Janeiro (Paleoceno superior: Itaboraiense). VIII Simpósio Brasileiro de Paleontologia de Vertebrados (Boletim de Resumos), 140.

Migotto, R. (2008) Inferência filogenética em gaviões buteoninos (Aves: Accipitridae), com base em caracteres osteológicos cranianos. Universidade de São Paulo, São Paulo, 99 pp.

Migotto, R. (2013) Filogenia de Accipitridae (Aves: Accipitriformes) com base em caracteres osteológicos. Universidade de São Paulo, São Paulo, 304 pp.

Migotto, R. \& Alvarenga, H. (2007) The diversity of condors and vultures (Aves: Vulturidae) in the South American Quaternary. Ameghiniana, 44 (4), Suplemento, 29R.

Miller, A.H. (1953) A fossil hoatzin from the Miocene of Colombia. The Auk, 70, 484489.

Miller, L. (1931) Bird remains from the Kern River, Pliocene of California. The Condor, 33 (2), 70-72.

Miller, L. (1932) The Pleistocene storks of California. The Condor, 34, 212-216. 
Milne-Edwards, A. (1869-1871) Recherches Anatomiques et Paléontologiques pour Servir a l'Histoire des Oiseaux Fossiles de la France. Tome Second. Libraire de G. Masson, Paris, 627 pp.

Mingatos, G.S. (2017) Caça ou Deixa Passar? A Dieta dos Grupos Humanos do Sítio Lapa do Santo, Lagoa Santa, Minas Gerais. Universidade Federal do Rio de Janeiro, Rio de Janeiro, 88 pp.

Mlíkovský, J. (2002) Cenozoic Birds of the World. Part 1: Europe. Ninox Press, Praha, $417 \mathrm{pp}$.

Mohr, B.A.R., Bernardes-de-Oliveira, M.E.C. \& Loveridge, R.F. (2007) The macrophyte flora of the Crato Formation. In: Martill, D.M., Bechly, G. \& Loveridge, R.F. (Eds.), The Crato Fossil Beds of Brazil: Window into an Ancient World. Cambridge University Press, Cambridge, pp. 537-565.

Mones, A. (1986) Palaeovertebrata Sudamericana. Catálogo Sistemático de los Vertebrados Fósiles de América del Sur. Parte I. Lista Preliminar y Bibliografía. Courier Forschungsinstitut Senckenberg, 82, 1-625.

Moon, P. (2012) O Colecionador de Taubaté. Época, 748 (17 September 2012), 72-76.

Moraes, T.G.C. (2014) Arqueofauna do sitio Toca da Janela da Barra do Antonião: um estudo sobre Paleoecologia Humana na Serra da Capivara. Universidade Federal do Vale do São Francisco, São Raimundo Nonato, 80 pp.

Moreno, F.P. (1889) Breve reseña de los progresos del Museo La Plata, durante el segundo semestre de 1888. Boletin del Museo La Plata (Provincia de Buenos Aires), $3,1-44$.

Moreno, F.P. \& Mercerat, A. (1891) Catálogo de los pájaros fósiles de la República Argentina conservados en el Museo de La Plata. Anales del Museo de La Plata, 1, 7 71.

Mortensen, T. (1925) Herluf Winge (1857-1923). Annual Report Smithsonian Institution, 1924, 513-522. 
Mourer-Chauviré, C. (Ed.) (1988) Society of Avian Paleontology and Evolution Information Letter $n^{\circ}$ 2, November 1988. SAPE, Villeurbanne.

Mourer-Chauviré, C. (Ed.) (1989) Society of Avian Paleontology and Evolution Information Letter $n^{\circ}$ 3, October 1989. SAPE, Villeurbanne.

Mourer-Chauviré, C. (1992a) The Galliformes (Aves) from the Phosphorites du Quercy (France): Systematics and Biostratigraphy. Natural History Museum of Los Angeles County, Science Series, 36, 67-95.

Mourer-Chauviré, C. (Ed.) (1992b) Society of Avian Paleontology and Evolution Information Letter $n^{\circ}$ 6, November 1992. SAPE, Villeurbanne.

Mourer-Chauviré, C. (1999) Les relations entre les avifaunes du Tertiaire inférieur d'Europe et d'Amérique du Sud. Bulletin de la Société Géologique de France, 170 (1), 85-90.

Mourer-Chauviré, C. (2000) A New Species of Ameripodius (Aves: Galliformes: Quercymegapodiidae) from the Lower Miocene of France. Palaeontology, 43 (3), 481-493.

Mourer-Chauviré, C. (2003) Birds (Aves) from the Middle Miocene of Arrisdrift (Namibia). Preliminary study with description of two new genera: Amanuensis (Accipitriformes, Sagittariidae) and Namibiavis (Gruiformes, Idiornithidae). Geological Survey of Namibia Memoir, 19, 103-113.

Mourer-Chauviré, C. (2004) Review of "Cenozoic Birds of the World, Part 1: Europe". The Auk, 121 (2), 623-627.

Mourer-Chauviré, C. (2006) The Avifauna of the Eocene and Oligocene Phosphorites du Quercy (France): An Updated List. Strata Série 1, 13, 135-149.

Mourer-Chauviré, C., Pickford, M. \& Senut, B. (2011) The first Palaeogene galliform from Africa. Journal of Ornithology, 152 (3), 617-622.

Murray, P.F. \& Vickers-Rich, P. (2004) Magnificent Mihirungs: the colossal flightless birds of the Australian dreamtime. Indiana University Press, Indiana, 416 pp. 
Museu de História Natural de Taubaté (MHNT) (undated A) Histórico. Available from: http://www.museuhistorianatural.com/historico.html (accessed 1 August 2018).

Museu de História Natural de Taubaté (MHNT) (undated B) Pesquisa. Available from: http://www.museuhistorianatural.com/pesquisa.html (accessed 1 August 2018).

Naish, D., Martill, D.M. \& Merrick, I. (2007) Birds of the Crato Formation. In: Martill, D.M., Bechly, G. \& Loveridge, R.F. (Eds.), The Crato Fossil Beds of Brazil: Window into an Ancient World. Cambridge University Press, Cambridge, pp. 525-533.

Nascimento, R. \& Silveira, L.F. (2020) The Fossil Birds of Peter Lund. Zootaxa, 4743 (4), 480-510.

Nascimento, R.S. \& Silveira, L.F. (2021) Aves Fósseis do Brasil. XXVII Congresso Brasileiro de Ornitologia (Livro de Resumos), 124.

Nascimento, T.H.M.S. \& Oliveira, G.R. (2018) Identificação e descrição de penas fósseis da Formação Crato, Cretáceo Inferior da Bacia do Araripe. Anais do XVIII JEPEX, 10367-1.

Nava, W.R. (2013) Vertebrados do afloramento "Enantiornithes", de Presidente Prudente, Grupo Bauru do Estado de São Paulo. Boletim de Resumos do XXIII Congresso Brasileiro de Paleontologia, Paleontologia em Destaque, Edição Especial (Outubro/2013), 253.

Nava, W.R. (2015) Vertebrados do afloramento "Enantiornithes", de Presidente Prudente, Grupo Bauru, Formação Adamantina do Estado de São Paulo. Paleo 2014, Súmula dos encontros e Resumos, Paleontologia em Destaque, 68, 138.

Nava, W.R., Alvarenga, H., Chiappe, L.M. \& Martinelli, A.G. (2015) Threedimensionally preserved cranial remains of enantiornithine birds from the Late Cretaceous of Brazil. Libro de resúmenes del V Congreso Latinoamericano de Paleontología de Vertebrados, 73.

Negri, F.R., Bocquentin-Villanueva, J., Ferigolo, J. \& Antoine, P.O. (2010) A review of Tertiary mammal faunas and birds from western Amazonia. In: Hoorn, C. \& 
Wesselingh, F.P. (Eds.) Amazonia: Landscape and Species Evolution, A Look into the Past. Wiley-Blackwell, Oxford, pp. 245-258.

Negri, F.R. \& Ferigolo, J. (1999) Anatomia craniana de Neoepiblema ambrosettianus (Ameghino, 1889) (Rodentia, Caviomorpha, Neoepiblemidae) do Mioceno SuperiorPlioceno, Estado do Acre, Brasil, e revisão das espécies do gênero. Boletim do Museu Paraense Emílio Goeldi, Série Ciências da Terra, 11, 1-81.

Newton, A. (1881) Hoactzin. In: The Encyclopadia Britannica: a Dictionary of Arts, Sciences, and General Literature. Ninth Edition. Volume XII. Charles Scribner's Sons, New York, pp. 28-29.

Noriega, J.I. (1992) Un nuevo genero de Anhingidae (Aves: Pelecaniformes) de la Formacion Ituzaingo (Mioceno Superior) de Argentina. Notas del Museo de La Plata, 21 (Paleontologia No 109), 217-223.

Noriega, J.I. (1995) The avifauna from the "Mesopotamian" (Ituzaingó Formation; Upper Miocene) of Entre Rios Province, Argentina. Courier Forschungsinstitut Senckenberg, 181, 141-148.

Noriega, J.I. \& Agnolin, F.L. (2008) El registro paleontológico de las Aves del "Mesopotamiense" (Formación Ituzaingó; Mioceno tardío-Plioceno) de la provincia de Entre Ríos, Argentina. INSUGEO, Miscelánea, 17 (2), 271-290.

Noriega, J.I. \& Alvarenga, H.M.F. (2002) Phylogeny of the Tertiary Giant Anhingas (Pelecaniformes: Anhingidae) from South America. Proceedings of the 5th Symposium of the Society of Avian Paleontology and Evolution, 41-49.

Novaes, L.S. (2013) Adaptação osteológica para a terrestrialidade em pica-paus (Aves: Picidae). Universidade de São Paulo, São Paulo, 65 pp.

O’Connor, J. (2020) The Plumage of Basal Birds. In: Foth, C. \& Rauhut, O.W.M. (Eds.), The Evolution of Feathers: From Their Origin to the Present. Springer, Cham, pp. 147-172. 
O’Connor, J.K., Falk, A., Wang, M. \& Zheng, X. (2020) First report of immature feathers in juvenile enantiornithines from the Early Cretaceous Jehol avifauna. Vertebrata PalAsiatica, 58 (1), 24-44.

O’Connor, J. \& Dyke, G. (2010) A Reassessment of Sinornis santensis and Cathayornis yandica (Aves: Enantiornithes). Records of the Australian Museum, 62 (1), 7-20.

Oberholser, H.C. (1918) Diagnosis of a new genus of Anatidae from South America. Journal of the Washington Academy of Sciences, 8, 571-572.

Oliveira, E.V. (1999) Quaternary vertebrates and climates from southern Brazil. In: Rabassa, J. \& Salemme, M. (Eds.) Quaternary of South America and Antarctic Peninsula Volume 12 (1996-1997). A. A. Balkema, Rotterdam, pp. 61-73.

Oliveira, I.P.M.R. (2008) Barramentos naturais no Vale do Rio Peruaçu: conseqüências ecológicas e ambientais de eventos pretéritos e futuros. Universidade Federal de Lavras, Lavras, 146 pp.

Oliveira, P.R.S., Donatelli, R.J., Neves, A.S., Santos, F.C.V., Santos, S.S., Nascimento, M.S., Ferreira, G.J.B.C. \& Guzzi, A. (2019) Compared Cranial Osteology of Species of Leptoptilus (Lesson, 1831) (Aves, Ciconiidae). Comunicata Scientiae, 10 (2), 232-243.

Oliveira, T.V., Scherer, C.S. \& Jesus, D.S. (2016) Morcegos quaternários na Toca dos Ossos, Bahia, Brasil: a importância de predadores na gênese de concentrações fossilíferas. Boletim de Resumos - X Simpósio Brasileiro de Paleontologia de Vertebrados, Paleontologia em Destaque, Edição Especial (Outubro/2016), 188.

Oliveira, Y.A.B., Silva, J.L.L., Silva, A.P.L., Oliveira, E.V. \& Silva, E.P.A. (2013) Primeiro registro de ave fóssil em depósito pleistocênico no estado de Alagoas, Brasil. Paleontologia em Destaque, Edição Especial (Outubro/2013), 259.

Olson, S.L. (1973) Evolution of the Rails of the South Atlantic Islands (Aves: Rallidae). Smithsonian Contributions to Zoology, 152, 1-53.

Olson, S.L. (1975) Paleornithology of St. Helena Island, South Atlantic Ocean. Smithsonian Contributions to Paleobiology, 23, 1-49. 
Olson, S.L. (1976) A Jacana from the Pliocene of Florida (Aves: Jacanidae). Proceedings of the Biological Society of Washington, 89 (19), 259-264.

Olson, S. L. (1977) A Synopsis of the Fossil Rallidae. In: Ripley, S.D. Rails of the World. David R. Godine, Boston, pp. 339-373.

Olson, S.L. (1981) Natural History of Vertebrates on the Brazilian Islands of the Mid South Atlantic. National Geographic Society Research Reports, 13, 481-492.

Olson, S.L. (1985a) Faunal turnover in South American fossil avifauna: the insufficiencies of the fossil record. Evolution, 39 (5), 1174-1177.

Olson, S.L. (1985b) The fossil record of birds. In: Farner, D.S., King, J.R. \& Parkes, K.C. (Eds.), Avian Biology. Volume 8. Academic Press, New York, pp. 79-252.

Olson, S.L. (2003) Development and uses of avian skeleton collections. Bulletin of the British Ornithologists' Club, 123A, 27-34.

Olson, S.L. \& Alvarenga, H.M.F. (2002) A new genus of small teratorn from the Middle Tertiary of the Taubaté Basin, Brazil (Aves: Teratornithidae). Proceedings of the Biological Society of Washington, 115 (4), 701-705.

Olson, S.L. \& Feduccia, A. (1980) Relationships and Evolution of Flamingos (Aves: Phoenicopteridae). Smithsonian Contributions to Zoology, 316, 1-73.

Orta, J., Garcia, E.F.J., Kirwan, G.M. \& Boesman, P. (2016). Lesser Frigatebird (Fregata ariel). In: del Hoyo, J., Elliott, A., Sargatal, J., Christie, D.A. \& de Juana, E. (Eds.) Handbook of the Birds of the World Alive. Lynx Edicions, Barcelona. Retrieved from: http://www.hbw.com/node/52671 (Accessed 9 February 2016).

Padberg Drenkpol, J.A. (1927) Um Benemerito do Brasil: O dinamarquês Herluf Winge classificador dos achados paleontologicos de Lund (1857-1923). Boletim do Museu Nacional do Rio de Janeiro, 3 (1), 1-14.

Padberg Drenkpol, J.A. (1931) Sobre a paleontologia dos papagaios. Boletim do Museu Nacional, 7 (3), 215-225. 
Palma, J.M.C. (1973) Paleontologia e estratigrafia da Bacia de São José de Itaboraí, Estado do Rio de Janeiro. Universidade Federal do Rio de Janeiro, Rio de Janeiro, $61 \mathrm{pp}$.

Palma, J.M.C. \& Brito, I.B. (1974) Paleontologia e Estratigrafia da Bacia de São José de Itaboraí, Estado do Rio de Janeiro. Anais da Academia Brasileira de Ciências, 46 (3/4), 383-406.

Pacheco, J.F., Silveira, L.F., Aleixo, A., Agne, C.E., Bencke, G.A., Bravo, G.A., Brito, G.R.R., Cohn-Haft, M., Maurício, G.N., Naka, L.N., Olmos, F., Posso, S.R., Lees, A.C., Figueiredo, L.F.A., Carrano, E., Guedes, R.C., Cesari, E., Franz, I., Schunck, F. \& Piacentini, V.Q. (2021) Annotated checklist of the birds of Brazil by the Brazilian Ornithological Records Committee-second edition. Ornithology Research, 29, 94-105.

Pascotto, M.C. \& Donatelli, R.J. (2003) Cranial Osteology in Momotidae (Aves: Coraciiformes). Journal of Morphology, 258 (1): 32-48.

Pascotto, M.C., Höfling, E. \& Donatelli, R.J. (2006a) Osteologia craniana de Coraciiformes (Aves). Revista Brasileira de Zoologia, 23 (3), 841-864.

Pascotto, M.C., Höfling, E. \& Donatelli, R.J. (2006b) The Ringed Kingfisher, Ceryle or Megaceryle torquata (Cerylinae, Alcedinidae, Coraciiformes)? An osteological view. Ornitologia Neotropical, 17 (4): 481-490.

Patterson, B. \& Kraglievich, J.L. (1960) Sistemáticas y Nomenclatura de las Aves Fororracoideas del Plioceno Argentino. Publicaciones del Museo Municipal de Ciencias Naturales y Tradicional de Mar de Plata, 1 (1), 1-52.

Patusco, T.B.S., Leonel, F.B.M., Machado, E.B., Araújo-Júnior, H.I., Metello, T.M. \& Kellner, A.W.A. (2016) Preliminary note on the first avian fossil record from the João Cativo Paleontological Site, a Quaternary tank deposit situated in Itapipoca, Ceará State, northeastern Brazil. X Simpósio Brasileiro de Paleontologia de Vertebrados (Boletim de Resumos), 190. 
Paula Couto, C. (Ed.) (1950) Memórias sobre a Paleontologia Brasileira. Instituto Nacional do Livro, Rio de Janeiro, 589 pp.

Paula Couto, C. (1953) Paleontologia Brasileira. Mamíferos. Instituto Nacional do Livro, Rio de Janeiro, 516 pp.

Paula Couto, C. (1958a) Idade geológica das bacias cenozóicas do Vale do Paraíba e Itaboraí. Boletim do Museu Nacional, Nova Série, Geologia, 25, 1-18.

Paula Couto, C. (1958b) Notas à margem de uma expedição científica a Minas Gerais. Kriterion, XI (45-46), 401-423.

Paula Couto, C. (1961) Relatório e prestação de contas de excursão. Museu Nacional, Rio de Janeiro, 15 pp. [unpublished]

Paula Couto, C. (1962) Explorações paleontológicas no Pleistoceno do Nordeste. Anais da Academia Brasileira de Ciências, 34 (3), XIX.

Paula Couto, C. (1970) Evolução de comunidades, modificações faunísticas e integrações biocenóticas dos vertebrados cenozóicos do Brasil. Actas IV Congresso Latino-Americano de Zoologia, 2, 907-930.

Paula Couto, C. (1980) Fossil Pleistocene to Sub-Recent Mammals From Northeastern Brazil. I - Edentata Megalonychidae. Anais da Academia Brasileira de Ciências, 52 (1), 143-151.

Paulo, P.O. (2009) Vertebrados fósseis do estado de Goiás, com ênfase em sua fauna de amniotas, compreendida entre o período permiano e a época pleistoceno. Universidade Estadual Paulista, Rio Claro, 165 pp.

Penido, I.S., Santos, L.V., Brandão, M.P., Vasconcelos, A.G. \& Kraemer, B.M. (2012) Primeiro registro de Geranospiza caerulescens (Falconiformes - Accipitridae) para o Quaternário do Brasil. Paleo Minas 2012 (Caderno de Resumos), 8.

Penido, I.S., Santos, L.V., Brandão, M.P., Vasconcelos, A.G. \& Kraemer, B.M. (2013) Primeiro registro de Geranospiza caerulescens (Falconiformes, Accipitridae) para o Quaternário do Brasil. Paleontologia em Destaque, 66, 116-117. 
Pereira, C.T., Rangel, C.C., Alves, D.S.J., Oliveira, E.F., Limonta, F.S., Ramalho, F.L., Souza, L.C.A., Alves, M.C.M., Castilho, L.H.A. \& Alves, E.J.F. (2015) Fósseis do Cretáceo na Região do Triângulo Mineiro: Lista Taxonômica. In: Candeiro, C.R.A. \& Avilla, L.S. (Org.) Fósseis de Vertebrados e Plantas do Período dos Dinossauros da Região do Triângulo Mineiro. Letra Capital, Rio de Janeiro, pp. 170-175.

Perez, C.P. (2009) Paleoecologia de mamíferos viventes como ferramenta na caracterização do ambiente holocênico de Lagoa Santa, MG. Universidade de São Paulo, São Paulo, 105 pp.

Perrichot, V., Marion, L., Néraudeau, D., Vullo, R. \& Tafforeau, P. (2008) The early evolution of feathers: fossil evidence from Cretaceous amber of France. Proceedings of the Royal Society B (Biological Sciences), 275 (1639), 1197-1202.

Pessis, A.M. (2003) Imagens da pré-historia. Parque Nacional Serra da Capivara. FUMDHAM/Petrobras, São Paulo, 308 pp.

Pessoa, P., Atman, D. \& Kimura, G. (2020) Environmental Problems in the Lagoa Santa Karst. In: Auler, A.S. \& Pessoa, P. (Eds.) Lagoa Santa Karst: Brazil's Iconic Karst Region. Springer, Cham, pp. 283-303.

Peters, D.S. (1988) Ein vollständiges Exemplar von Palaeotis weigelti (Aves, Palaeognathae). Courier Forschungsinstitut Senckenberg, 107, 223-233.

Peters, D.S. \& Storch, G. (1993) South American Relationships of Messel Birds and Mammals. Kaupia, 3, 263-269.

Pinheiro, A.E., Castro, L.O.R., Pereira, P.V.L.G.C., Bergqvist, L.P., Bertolossi, M.L. P. \& Silva, K.F. (2018) Primeiro registro de um réptil de grandes proporções para a Bacia de Itaboraí, Rio de Janeiro. XI Simpósio Brasileiro de Paleontologia de Vertebrados (Boletim de Resumos), 88.

Pinheiro, A.E.P., Bertolossi, M.L.P., Silva, K.F., Paraízo, N.F., Carvalho, L.S., Madeira, R.O.A., Calabrot, G.S.C., Oliveira, L.L.R., Nascimento, R.L., Garuba, C.P., Maia, B.M.S, Bandeira, K.L.N., Pereira, P.V.L.G.C., Brum, A.S., Nunes, F.R. \& Souza, L.G. (2019) Atividades de extensão educacional da FFP/UERJ, um complemento ao 
campo de paleontologia. XXVI Congresso Brasileiro de Paleontologia (Boletim de Resumos), Paleontologia em Destaque (Edição especial, Outubro de 2019), 160.

Pinheiro, F.L. \& Fernandes-Ferreira, H. (2014) História da Zoologia no Estado do Ceará. Parte II: Paleozoologia e Etnozoologia. Gaia Scientia, 8 (1), 121-135.

Pittman, M., O’Connor, J., Tse, E., Makovicky, P., Field, D.J., Ma, W., Turner, A.H., Norell, M.A., Pei, R. \& Xu, X. (2020) The Fossil Record of Mesozoic and Paleocene Pennaraptorans. Bulletin of the American Museum of Natural History, 440, 37-95.

Pivetta, M. (2003) Asas do passado. Pesquisa FAPESP, 93, 52-55.

Pivetta, M. (2011) A longa viagem da cigana. Pesquisa FAPESP, 189, 48-51.

Porto, M. (2004) Anatomia comparada do esqueleto da cabeça e da musculatura da mastigação de Anodorhynchus Spix, 1824, Ara Lacépède, 1799, Diopsittaca Ridgway, 1912, Prophyrrura Miranda-Ribeiro, 1920 e Orthopsittaca Ridgway, 1912 (Aves: Psittaciformes: Arinae). Universidade Federal Rural do Rio de Janeiro, Seropédica, 88 pp.

Posso, S.R. \& Donatelli, R.J. (2001) Cranial osteology and systematic implications in Crotophaginae (Aves, Cuculidae). Journal of Zoological Systematics and Evolutionary Research, 39 (4), 247-256.

Posso, S.R. \& Donatelli, R.J. (2005) Skull and Mandible Formation in the Cuckoo (Aves, Cuculidae): Contributions to the nomenclature in avian osteology and systematics. European Journal of Morphology, 42 (4/5), 163-172.

Posso, S.R. \& Donatelli, R.J. (2006) Análise filogenética e implicações sistemáticas evolutivas nos Cuculiformes (Aves) com base na osteologia, comportamento e ecologia. Revista Brasileira de Zoologia, 23 (3), 608-629.

Posso, S.R. \& Donatelli, R.J. (2007) Osteologia craniana e considerações sistemáticas em Coccyzinae (Cuculidae). Boletim do Museu Paraense Emílio Goeldi, Ciências Naturais, 2 (1), 87-116. 
Posso, S.R. \& Donatelli, R.J. (2010) When decisions on homologous structures cause ambiguous taxa relationships: the Neomorphinae (Aves, Cuculidae) example. Brazilian Journal of Biology, 70 (1), 195-204.

Posso, S.R., Donatelli, R.J., Piacentini, V.Q. \& Guzzi, A. (2020) Phylogeny and classification of the Bucconidae (Aves, Galbuliformes) based on osteological characters. Papéis Avulsos de Zoologia, v.60, e20206027, 1-20.

Prado, G.M.E.M. \& Anelli, L.E. (2013) Dois novos registros de penas fósseis da Formação Santana, Bacia do Araripe, Nordeste do Brasil. Paleontologia em Destaque, Edição Especial (Outubro/2013), 266.

Prado, G.M.E.M, \& Anelli, L.E. (2015) Dark dinosaurs: colour and environment of the Cretaceous and Paleogene of Brazil. Paleo 2014, Súmula dos encontros e Resumos, Paleontologia em Destaque, 68, 148.

Prado, G.M.E.M., Anelli, L.E., Pacheco, M.L.A.F., Petri, S. \& Romero, G.R. (2016b) Systematic and taphonomic insights of fossilized feathers: A new occurrence from the Oligocene of Taubaté Basin (SE, Brazil). Journal of South American Earth Sciences, 72, 169-177.

Prado, G.M.E.M., Anelli, L.E., Petri, S. \& Romero, G.R. (2016a) New occurrences of fossilized feathers: systematics and taphonomy of the Santana Formation of the Araripe Basin (Cretaceous), NE, Brazil. PeerJ, 4:e1916, 1-31.

Prefeitura de Marília (2018) Museu de Paleontologia recebe pesquisadoras do Museu de História Natural de Los Angeles. Prefeitura de Marília. Available from: https://www.marilia.sp.gov.br/portal/noticias/0/3/1799/museu-de-paleontologiarecebe-pesquisadoras-do-museu-de-historia-natural-de-los-angeles (accessed 31 May 2018).

Prefeitura de Presidente Prudente (2020) Cercamento com alambrado visa proteger área de sítio paleontológico. Prefeitura de Presidente Prudente. Available from: http://www.presidenteprudente.sp.gov.br/site/noticias.xhtml?cod=48751 (accessed 28 July 2020). 
Previatto, D.M. (2012) Osteologia craniana da família Anhimidae (Aves: Anseriformes). Universidade Estadual Paulista, Botucatu, 99 pp.

Previatto, D.M. \& Posso, S.R. (2015) Cranial osteology of Cyclarhis gujanensis (Aves: Vireonidae). Papéis Avulsos de Zoologia, 55 (18), 255-260.

Proctor, H.C. (2003) Feather Mites (Acari: Astigmata): Ecology, Behaviour, and Evolution. Annual Review of Entomology, 48, 185-209.

Prous, A. (2016) As Missões arqueológicas desenvolvidas na região de Lagoa Santa na segunda Metade do século XX. In: Da-Gloria, P., Neves, W.A. \& Hubbe, M. (Org.), Lagoa Santa: história das pesquisas arqueológicas e paleontológicas. Annablume Arqueológica, São Paulo, pp. 111-130.

Prum, R.O. (1999) Development and Evolutionary Origin of Feathers. Journal of Experimental Zoology (Molecular and Developmental Evolution), 285 (4), 291-306.

Prum, R.O. \& Brush, A.H. (2002) The evolutionary origin and diversification of feathers. Quarterly Review of Biology, 77 (3), 261-295.

Raczka, M.F., Bush, M.B. \& Oliveira, P.E. (2018) The collapse of megafaunal populations in southeastern Brazil. Quaternary Research, 89 (1), 103-118.

Raczka, M.F., Oliveira, P.E., Bush, M. \& McMichael, C.H. (2013) Two paleoecological histories spanning the period of human settlement in southeastern Brazil. Journal of Quaternary Science, 28 (2), 144-151.

Rasmussen, D.T. \& Kay, R.F. (1992) A Miocene anhinga from Colombia, and comments on the zoogeographic relationships of South America's Tertiary avifauna. Natural History Museum of Los Angeles Science Series, 36, 225-230.

Rédua, L.S., Marinho, T.S., Martinelli, A.G. \& Ribeiro, L.C.B. (2016) Aspectos paleoecológicos do Sítio Paleontológico de Peirópolis (Maastrichtiano), Uberaba (MG). X Simpósio Brasileiro de Paleontologia de Vertebrados (Boletim de Resumos), 145. 
Reinhardt, J. (1867) De brasilianske Knoglehuler og de i dem forekommende Dyrelevninger. Fire Foredrag i den naturhistoriske Forenings Søndagsmøder: III-IV. Tidsskrift for Populare Fremstillinger af Naturvidenskaben, Tredie række, fjerde binds, fjerde hefte, 291-353.

Reinhardt, J. (1870) Bidrag til Kundskab om Fuglefaunaen i Brasiliens Campos. Videnskabelige Meddelelser fra den naturhistoriske Forening i Kjöbenhavn, 1870 (1-7), 1-124; 1870 (21-28), 315-457.

Reinhardt, J. (1881) Om de formentlige Levninger af en kæmpemæssig, med Cariama beslægtet, uddød Fugl fra Brasiliens Knoglehuler. Videnskabelige Meddelelser fra den naturhistoriske Forening i Kjфbenhavn, 1881 [1882], 141-153.

Reinhardt, J. (1882) On the Remains of an extinct gigantic Bird supposed to be allied to Cariama, from the Ossiferous Caves of Brazil. The Ibis, 24 (2), 321-332.

Reis, V.G.M., Fernandes, A.C.S. \& Carvalho, I.S. (2014) Ovos fósseis do Cretáceo do Brasil. Anais do $47^{\circ}$ Congresso Brasileiro de Geologia, 184.

Ribeiro, A.M., Alvarenga, H.M. \& Rosenau, M. (1995) Primeiro registro de ave fóssil para a Formação Touro Passo (Pleistoceno superior-Holoceno inferior) do Rio Grande do Sul. Atas do XIV Congresso Brasileiro de Paleontologia, 107.

Rice, N.H. (2005) Further Evidence for Paraphyly of the Formicariidae (Passeriformes). The Condor, 107, 910-915.

Rich, P.V. (1979) Fossil Birds of Old Gondwanaland: A Comment on Drifting Continents and Their Passengers. In: Gray, J. \& Boucot, A.J. (Eds.) Historical Biogeography, Plate Tectonics and the Changing Environment. Proceedings of the Thirty-seventh Annual Biology Colloquium and Selected Papers. Oregon State University Press, Corvallis, pp. 321-332.

Riff, D., Mader, B., Kellner, A.W.A. \& Russell, D. (2004) An Avian Vertebra from the Continental Cretaceous of Morocco, Africa. Arquivos do Museu Nacional, 62 (2), $217-223$. 
Rincón, A.D. \& Stucchi, M. (2003) Primer registro de familia Pelagornithidae (Aves: Pelecaniformes) para Venezuela. Boletin de la Sociedad Venezoelana de Espeleologia, 37, 27-30.

Rinderknecht, A. \& Noriega, J.I. (2002) Un nuevo género de Anhingidae (Aves: Pelecaniformes) del Plioceno-Pleistoceno del Uruguay (Formación San José). Ameghiniana, 39 (2), 183-191.

Rodrigues, I., Castro, F., Deantoni, F.O., Carvalho, L.B., Bianchini, G.F. \& Gaspar, M.D. (2013) Primeiro registro de Cheloniidae (Testudines, Cryptodira) para o Sambaqui de Sernambetiba (Recôncavo da Baía de Guanabara, RJ). Paleontologia em Destaque, Edição Especial (Outubro/2013), 273.

Rodrigues, M. (2008) Noteworthy Bird records at Lagoa Santa, southeastern Brazil. Revista Brasileira de Zoologia, 25 (1), 150-153.

Roosevelt, A.C., Lima da Costa, M., Lopes Machado, C., Michab, M., Mercier, N., Valladas, H., Feathers, J., Barnett, W., Imazio da Silveira, M., Henderson, A., Sliva, J., Chernoff, B., Reese, D.S., Holman, J.A., Toth, N. \& Schick, K. (1996) Paleoindian Cave Dwellers in the Amazon: The Peopling of the Americas. Science, $272,373-384$.

Rothschild, W. (1907) Extinct Birds. Hutchison \& Co., London, 244 pp.

Roy, A., Rogers, C.S., Clements, T., Pittman, M., Habimana, O., Martin, P. \& Vinther, J. (2020) Fossil Microbodies Are Melanosomes: Evaluating and Rejecting the "Fossilized Decay-Associated Microbes" Hypothesis. Bulletin of the American Museum of Natural History, 440, 251-276.

Ruschi, A. (1979) Aves do Brasil. Editora Rios, São Paulo, 335 pp.

Sales, H.R. (2003) Fossilização de Aves na Lapa do Rezar, Parque Nacional Cavernas do Peruaçu, Minas Gerais, Brasil. Anais do XXVII Congresso Brasileiro de Espeleologia. Sociedade Brasileira de Espeleologia, 212-215.

Salomão, C.C. (2015) Osteologia e filogenia de Asio Brisson, 1760 (Strigiformes, Strigidae). Universidade Federal de Mato Grosso do Sul, Campo Grande, 72 pp. 
Salum, L., Acosta Hospitaleche, C., Avilla, L.S. (2016) Os primeiros registros fossilíferos de Passariformes (Dinosauria: Aves) da Gruta dos Ursos, Estado do Tocantins, Norte do Brasil. Boletim de Resumos - X Simpósio Brasileiro de Paleontologia de Vertebrados, Paleontologia em Destaque, Edição Especial (Outubro/ 2016), 153.

Santos, M. (1999) Serra da Mantiqueira e Planalto do Alto Rio Grande: a bacia terciária de Aiuruoca e evolução morfotectônica. Universidade Estadual Paulista, Rio Claro, 2 vol., 134 pp.

Santos, S.S., Nobushige, S.Y.L., Ribeiro, A.S.N., Santos, F.C.V, Donatelli, R.J., Ferreira, G.J.B.C. \& Guzzi, A. (2018) Phylogeny of the species of Ciconia (Aves, Ciconiidae) based on cranial osteological characteristics. Comunicata Scientiae, 9 (4), 575-589.

SAPE (2002) Society of Avian Paleontology and Evolution Newsletter Letter $n^{o} 16$, October 2002. SAPE: Frankfurt am Main.

SAPE (2020) Society of Avian Paleontology and Evolution Newsletter Letter $n^{o} 34$, November 2020. SAPE: Christchurch.

Sayão, J.M., Saraiva, A.A.F. \& Uejima, A.M.K. (2011) New evidence of feathers in the Crato Formation supporting a reappraisal on the presence of Aves. Anais da Academia Brasileira de Ciências, 83 (1), 197-210.

Sayão, J.M. \& Uejima, A.M.K. (2009) Novos registros de penas isoladas na Formação Crato, Grupo Santana, Bacia do Araripe, nordeste do Brasil. Paleo 2009 (Resumos), 22.

Sayão, J.M. \& Uejima, A.M.K. (2010) Novos registros de penas isoladas na Formação Crato, Grupo Santana, Bacia do Araripe, nordeste do Brasil. Paleontologia em Desataque, 63, 54.

Schäff, Dr. (1888) Die Vögel aus den Knochenhöhlen in Brasilien. Journal für Ornithologie, 36 (181), 5-8.

Sclater, P.L. \& Saunders, H. (Eds.) (1888) The Ibis, 30 (2), pp. 282. 
Schmitz, P.I. (1990) O povoamento pleistocênico do Brasil. Revista Arqueología Americana, 1, 33-68.

Sedor, F.A., Oliveira, E.V., Silva, D.D., Fernandes, L.A., Cunha, R.F., Ribeiro, A.M. \& Dias, E.V. (2014a) A new South American Paleogene fauna, Guabirotuba Formation (Curitiba, Paraná state, South of Brazil). Abstract Volume - 4th International Palaeontological Congress, 614.

Sedor, F.A., Dias, E.V., Cunha, R.F. \& Alvarenga, H. (2014b) Paleogene phorusrhacid bird (Aves, Phorusrhacidae) from the Guabirotuba Formation, Curitiba Basin, Paraná, South of Brazil. Abstract Volume - 4th International Palaeontological Congress, 807.

Sedor, F.A., Oliveira, E.V., Silva, D.D., Fernandes, L.A., Cunha, R.F., Ribeiro, A.M. \& Dias, E.V. (2016) A New South American Paleogene Land Mammal Fauna, Guabirotuba Formation (Southern Brazil). Journal of Mammalian Evolution, 24 (1), $39-55$.

Shufeldt, R.W. (1916) A Fossil Feather from Taubaté. The Auk, 33 (2), 206-207.

Sick, H. (1984a) Ornitologia Brasileira. Volume I. Editora Universidade de Brasília, Brasília, pp. 1-481.

Sick, H. (1984b) Ornitologia Brasileira. Volume II. Editora Universidade de Brasília, Brasília, pp. 482-827.

Sick, H. (1993) Birds in Brazil: A Natural History. Princeton University Press, Princeton, $703 \mathrm{pp}$

Sick, H. (1997) Ornitologia Brasileira. Editora Nova Fronteira, Rio de Janeiro, 912 pp.

Silva, A.G., Ferreira, G.J.B., Donatelli, R.J. \& Guzzi, A. (2012a) Osteologia craniana de Micrastur semitorquatus Vieillot, 1817 (Falconiformes: Falconidae). Comunicata Scientiae, 3 (1): 64-71.

Silva, A.G.C., Parallada, C.I. \& Melo, M.S. (2007) Pinturas rupestres do sítio arqueológico Abrigo Usina São Jorge, Ponta Grossa, Paraná. Revista Publicatio 
UEPG - Ciências Exatas e da Terra, Ciências Agrárias e Engenharias, 13 (1), 2533.

Silva, C.M. (2010). O registro de aves fósseis quaternárias da Toca da Boa Vista (Bahia-Brasil). Pontifícia Universidade Católica de Minas Gerais, Belo Horizonte.

Silva, C.M. \& Cozzuol, M.A. (2010) Aves fósseis da Toca da Boa Vista (PleistocenoHoloceno, Bahia, Brasil). $7^{o}$ Simpósio Brasileiro de Paleontologia de Vertebrados (Boletim de Resumos), 111.

Silva, C.M.; Penido, I.S. \& Vasconcelos, M.F. (2012b) Primeiro registro de Nyctibius griseus (Caprimulgiformes: Nyctibidae) fóssil para a Gruta dos Brejões (Bahia, Brasil). Anais do XIX Congresso Brasileiro de Ornitologia, 368.

Silva, D. (2011) Considerações taxonômicas em Ardeidae (Aves), com base na osteologia. Universidade de São Paulo, São Paulo, 47 pp.

Silva, J.L.L., Oliveira, Y.A.B., Silva, A.P.L., Omena, E.C. \& Silva, E.P.A. (2013) Primeiro registro de Eremotherium laurillardi prenhe em jazigo fossilífero pleistocênico, Alagoas, nordeste do Brasil. Paleontologia em Destaque, Edição Especial (Outubro/2013), 281-282.

Silva, R.R.N. (2014) Análise zooarqueológica das interações cinergéticas no sítio arqueológico Pedra do Alexandre, Rio Grande do Norte. IX Simpósio Brasileiro de Paleontologia de Vertebrados (Boletim de Resumos), 133.

Silva Santos, R. (1950) Vestígio de Ave Fóssil nos Folhelhos Betuminosos de Tremembé, S. Paulo. Anais da Academia Brasileira de Ciências, 22, 445-446.

Silveira, L.F. (2003) Filogenia dos Cracidae (Aves: Galliformes), com base em caracteres osteológicos. Universidade de São Paulo, São Paulo, 212 pp.

Silveira, L.F. \& Höfling, E. (2007) Osteologia craniana dos Tinamidae (Aves: Tinamiformes), com implicações sistemáticas. Boletim do Museu Paraense Emílio Goeldi, Ciências Naturais, 2 (1), 15-54. 
Silveira, L.F., Soares, E.S. \& Bianchi, C.A. (2008) Plano de Ação Nacional para a Conservação de Galliformes Ameaçados de Extinção (acaruãs, jacus, jacutingas, mutuns e urus). Série Espécies Ameaçadas - $n^{\circ}$ 6. ICMBio, Brasília, 90 pp.

Simões, P.R. (2001) Carste e Paleoecologia em São Raimundo Nonato - PI, Brasil. 13th International Congress of Speleology, 305-309.

Smithwick, F. \& Vinther, J. (2020) Palaeocolour: A History and State of the Art. In: Foth, C. \& Rauhut, O.W.M. (Eds.), The Evolution of Feathers: From Their Origin to the Present. Springer, Cham, pp. 185-211.

Smyth, R.S.H., Martill, D.M., Frey, E., Rivera-Sylva, H.E. \& Lenz, N. (2020) A maned theropod dinosaur from Gondwana with elaborate integumentary structures. Cretaceous Research, DOI: https://doi.org/10.1016/j.cretres.2020.104686

Soares, P.C., Landim, P.M.B, Fúlfaro, V.J. \& Sobreiro Neto, A.F. (1980) Ensaio de caracterização estratigráfica do Cretáceo no Estado de São Paulo: Grupo Bauru. Revista Brasileira de Geociências, 10, 177-185.

Solórzano, A. \& Rincón, A.D. (2015) The earliest record (early Miocene) of a bonytoothed bird from South America and a reexamination of Venezuelan pelagornithids. Journal of Vertebrate Paleontology, 35 (6), e995188, 1-8.

Soria, M.F. \& Alvarenga, H.M.F. (1989) Nuevos Restos de Mamíferos de la Cuenca de Taubaté, Estado de São Paulo, Brasil. Anais da Academia Brasileira de Ciências, 61 (2), 157-175.

Souto, A.A. \& Carvalho, L.B. (2010) Primeiro registro de fósseis de Anura (Amphibia) para a localidade de João Cativo (Itapipoca, CE), Pleistoceno ao sub-recente. $7^{o}$ Simpósio Brasileiro de Paleontologia de Vertebrados (Boletim de Resumos), 116.

Souto, P.R.F. (Org.) (2017) Icnologia de Paleovertebrados. Letra Capital, Rio de Janeiro, 198 pp.

Souza, M.S. \& Medeiros, O. (1982) Inscrições rupestres no Rio Grande do Norte. Coleção Textos Acadêmicos, Universidade Federal do Rio Grande do Norte, 214, 147. 
Souza Cunha, F.L. (1961) Relatório de excursão ao Nordeste. Museu Nacional, Rio de Janeiro, 8 pp. [unpublished]

Souza Cunha, F.L. (1962) Explorações paleontológicas no Pleistoceno do Rio Grande do Norte. Universidade do Estado da Guanabara, Rio de Janeiro, 51 pp.

Souza Cunha, F.L. (1966) Explorações paleontológicas no Pleistoceno do Rio Grande do Norte. Arquivos do Instituto de Antropologia "Câmara Cascudo", 2 (1-2), 75116.

Souza Cunha, F.L. (1978) Explorações paleontológicas no Pleistoceno do Rio Grande do Norte. Coleção Mossoroense C, 70, 1-112.

Souza Cunha, F.L. \& Guimarães, M.L. (1978) A fauna sub-recente de vertebrados do "Grande Abrigo da Lapa Vermelha (P.L.)" de Pedro Leopoldo, Minas Gerais. Arquivos do Museu de História Natural-UFMG, 3, 201-231.

Souza Cunha, F.L. \& Guimarães, M.L. (1981-1982) A fauna sub-recente de vertebrados do "Grande Abrigo da Lapa Vermelha Emperaire (P.L.)" Pedro Leopoldo, Estado de Minas Gerais. Revista do Museu Paulista, Nova Série, 28, 235-272.

Souza-Cunha, F.L. \& Magalhães, R.M.M. (1986) A fauna de vertebrados sub-recentes de Cerca Grande, Matozinhos, Minas Gerais. Anais do VI Congresso Brasileiro de Zoologia. Publicações avulsas do Museu Nacional, 65, 119-131.

Souza Cunha, F.L., Magalhães, R.M.M. \& Garcia, S. (1977) Anexo A - Vertebrados do Sambaqui do Forte. Revista do Museu Paulista, Série de Arqueologia, 5, 143-150.

Souza-Filho, J.P. \& Guilherme, E. (2015) A Paleontologia no Estado do Acre. Paleontologia no Estado do Acre. In: Adamy, A. (Ed.) Geodiversidade do Estado do Acre. CPRM, Porto Velho, pp. 147-158.

Spix, J.B. (1824) Avium species novae, quas in itinere annis MDCCCXVII-MDCCCXX per Brasiliam. Tomus 1. Munich: Hübschmanni.

Spulski, B. (1910) Odontopteryx longirostris n. sp. Zeitschrift der Deutschen Geologischen Gesellschaft, 62, 507-521. 
Stager, K.E. (1964) The role of olfaction in food location by the turkey vulture (Cathartes aura). Los Angeles County Museum Contributions in Science, 81, 1-63.

Steadman, D.W., Oswald, J.A. \& Rincón, A.D. (2015) The diversity and biogeography of late Pleistocene birds from the lowland Neotropics. Quaternary Research, 83 (3), $555-564$

Stefano, W., Bezerra, E.C.M., Araujo, F.M. \& Reis, M.I. (2012) As aves que Peter Wilhelm Lund não descreveu. Boletim de História e Filosofia da Biologia, 6 (4), 57.

Stejneger, L. (1885) Natural history of birds. In: Kingsley, J.S. (Ed.), The Standard Natural History 4. S.E. Cassino and Company, Boston, pp. 1-558.

Storer, R.W. (1960) The Classification of Birds. In: Marshall, A.J. (Ed.) (1960) Biology and Comparative Physiology of Birds, Volume 1. New York, Academic Press, pp; 57-93.

Suárez, W. \& Olson, S.L. (2003) New records of storks (Ciconiidae) from Quaternary asphalt deposits in Cuba. The Condor, 105, 150-154.

Talent, J.A., Duncan, P.M. \& Handby, P.L. (1966) Early Cretaceous Feathers from Victoria. Emu, 64 (2), 81-86.

Tambussi, C.P. (1995) The Fossil Rheiformes from Argentina. Courier Forschungsinstitut Senckenberg, 181, 121-129.

Tambussi, C.P. \& Degrange, F.J. (2013) South American and Antarctic Continental Cenozoic Birds: Paleobiogeographic Affinities and Disparities. Springer, Dordrecht. $113 \mathrm{pp}$.

Tambussi, C. \& Noriega, J. (1996) Summary of the Avian Fossil Record from Southern South America. Münchner Geowissenschaftliche Abhandlungen, 30, 245-264.

Taranto, R.C. (2012) Postura locomotora de Diogenornis fragilis (Aves: Rheiformes), da Bacia de São José de Itaboraí (Itaboraiense), Estado do Rio de Janeiro. Universidade Federal do Rio de Janeiro, Rio de Janeiro, 78 pp. 
Taranto, R.C. \& Bergqvist, L.P. (2009) Novos materiais (Aves) da bacia de São José de Itaboraí (Neopaleoceno), Rio de Janeiro, Brasil. Resúmenes de la XXIV Jornadas Argentinas de Paleontología de Vertebrados (Resúmenes). Ameghiniana, 46 (4), Suplemento, 51R.

Taranto, R.C. \& Bergqvist, L.P. (2010) Contribuição ao conhecimento das aves fósseis da Bacia de S. J. de Itaboraí: descrição de novos espécimes e cálculo da massa corpórea. $7^{o}$ Simpósio Brasileiro de Paleontologia de Vertebrados (Boletim de Resumos), 118.

Taranto, R.C., Bergqvist, L.P. \& Alvarenga, H.M.F. (2009a) Novos Ralliformes (AVES) da Bacia de São José de Itaboraí (Neopaleoceno), Rio de Janeiro, Brasil. XXXI Jornada Giulio Massarani de Iniciação Científica, Artística e Cultural UFRJ (Livro de resumos), 287.

Taranto, R.C., Bergqvist, L.P. \& Alvarenga, H.M.F. (2009) Biodiversidade das Aves Fósseis do Brasil. In: XXXI Jornada Giulio Massarani de Iniciação Científica, Artística e Cultural UFRJ (Livro de Resumos). Universidade Federal do Rio de Janeiro, Rio de Janeiro, pp. 380.

Taranto, R.C., Furtado, M.R., Lopes, L., Alves, Y.M. \& Pinheiro, A.E.P. (2010) Novas ocorrências de dentes arcossaurianos provenientes da Formação Presidente Prudente, Noroeste do Estado de São Paulo. Paleo Minas 2010 (Caderno de Resumos), 23.

Taranto, R.C., Loguercio, M.F., Bergqvist, L.P. \& Rocha-Barbosa, O. (2011) Comparative analysis of the hindlimb morphology of Diogenornis fragilis (Aves, Ratites - Paleocene) and flightless extant and extinct birds. IV Congreso Latinoamericano de Paleontología de Vertebrados (Resúmenes). Ameghiniana, 48 (4), Suplemento, R58-R59.

Távora, V.A., Santos, A.A.R. \& Araújo, R.N. (2010) Localidades fossilíferas da Formação Pirabas (Mioceno Inferior). Boletim do Museu Paraense Emílio Goeldi, Ciências Naturais, 5 (2), 207-224.

Taylor, B. (1996) Inaccessible Rail (Atlantisia rogersi). In: del Hoyo, J., Elliott, A., Sargatal, J., Christie, D.A. \& de Juana, E. (eds.) (2014). Handbook of the Birds of the 
World Alive. Lynx Edicions, Barcelona. Retrieved from: http://www.hbw.com/node/53636 (accessed 27 July 2015).

Taylor, B. \& Christie, D.A. (1996) Mangrove Rail (Rallus longirostris). In: del Hoyo, J., Elliott, A., Sargatal, J., Christie, D.A. \& de Juana, E. (Eds.) (2014) Handbook of the Birds of the World Alive. Lynx Edicions, Barcelona. Retrieved from: http://www.hbw.com/node/53619 (accessed 13 August 2015).

Teixeira, M.F. \& Saraiva, A.A.F. (2010) Coleta sistemática de fósseis na Formação Crato - Bacia do Araripe. Paleo 2010 Nordeste (Resumos), 46.

Thomé, C. (2005) Brasileiros acham aves de 80 milhões de anos. O Estado de S.Paulo, 11/ago/2005, A19.

Tomazela, J.M. (2019) Fósseis de aves com dentes da época dos dinossauros são achados no interior de SP. $O$ Estado de S. Paulo. Available from: https://ciencia.estadao.com.br/noticias/geral,fosseis-de-aves-com-dentes-da-epocados-dinossauros-sao-achados-no-interior-de-sp,70002849210 (accessed 30 May 2019).

Tonni, E.P. \& Laza, J.H. (1980) Las aves de la fauna local Paso de Otero (Pleistoceno tardio) de la Provincia de Buenos Aires. Su significacion ecologica, climatica y zoogeografica. Ameghiniana, 17 (4), 313-322.

Tordoff, H.B. (1959) A condor from the Upper Pliocene of Kansas. The Condor, 61 (5), $338-343$.

Tuffani, M. (2002) Fósseis vão do quintal para o museu. Galileu, 134. Avaiable from: http://revistagalileu.globo.com/Galileu/0,6993,ECT377629-1719,00.html (accessed 28 July 2018).

Valle, C. \& Carnevalli, N. (1973) O papel das corujas na interpretação de Lund. SOM, $5,8-9$.

Veloso, T.P.G. (1983) Estudo do material zoológico coletado nas escavações do Setor de Arqueologia da UFMG. In: X Congresso Brasileiro de Zoologia (Resumos). 
Sociedade Brasileira de Zoologia, Universidade Federal de Minas Gerais, Belo Horizonte, pp. 439-440.

Veloso, T.P.G. \& Resende, E.M.T.P. (1992) Vestígios alimentares nos sítios arqueológicos sob abrigos de Minas Gerais. In: Anais do $3^{\circ}$ Congresso da Associação Brasileira de Estudos do Quaternário. Associação Brasileira de Estudos do Quaternário, Belo Horizonte, pp. 389-414.

Venceslau, P.T. (2014) Herculano Alvarenga: Cientista Sonhador. Contato, 628, 6-7.

Vialou, A.V. (2005) Indústria Lítica. In: Vialou, A.V. (Ed.), Pré-história do Mato Grosso. Volume 1: Santa Elina. Edusp, São Paulo, pp. 167-176.

Vidal, F.W.H. \& Campos, D.A. (2008) Explotando calcário e salvando fósseis na Chapada do Araripe. Anais do III Congresso Brasileiro de Rochas Ornamentais e VI Simpósio de Rochas Ornamentais do Nordeste, 306-316.

Vinther, J., Briggs, D.E.G., Prum, R.O. \& Saranathan, V. (2008) The colour of fossil feathers. Biology Letters, 4 (5), 522-525.

Vogel, M.A.C. (1987) Restos de Vertebrados do Sambaqui Zé Espinho. In: Kneip, L.M. (Coord.) Coletores e Pescadores Pré-Históricos de Guaratiba Rio de Janeiro. UFRJ, Rio de Janeiro, 256 pp.

Vucetich, M.G., Cunha, F.L.S. \& Alvarenga, H.M.F. (1993) Un Roedor Caviomorpha de la Formación Tremembé (Cuenca de Taubaté), Estado de São Paulo, Brasil. Anais da Academia Brasileira de Ciências, 65 (3), 247-251.

Waldherr, F.R., Araújo Júnior, H.I. \& Rodrigues, S.W.O. (2017) Origem e morfologia dos tanques naturais do Nordeste do Brasil. Pesquisas em Geociências, 44 (3), 467488.

Waldherr, F.R., Araújo Júnior, H.I., Rodrigues, S.W.O. \& Ximenes, C.L. (2019) La importancia de los tanques naturales (mega gnammas) en la preservación de fósiles de la Megafauna cuaternaria en el Noreste de Brasil. Cadernos do Laboratorio Xeolóxico de Laxe, 41, 99-122. 
Wall, R., Alvarenga, H.M.F., Marshall, L.G. \& Salinas, P. (1991) Hallazgo del primer ave fósil del Terciario de Chile: un ánade (Pelecaniformes; Anhingidae), preservado en un ambiente deltaico-fluvial del Mioceno de Lonquimay, región de la Araucanía, Chile. Congreso Geológico Chileno 5 (Resúmenes Expandidos), 394-397.

Wallace, A.R. (1876) The geographical distribution of animals. Vol. I. Harper \& Brothers, New York, 503 pp.

Walter, H.V. (1958) Arqueologia da região de Lagoa Santa (Minas Gerais): índios précolombianos dos abrigos rochedos. Sedegra, Rio de Janeiro, 227 pp.

Weick, F. (2006) Owls (Strigiformes). Annotated and illustrated Checklist. Springer, Berlin, 388 pp.

Wing, L. W. (1956) Natural History of Birds: A Guide to Ornithology. The Ronald Press Company, New York, 539 pp.

Winge, O. (1887) Fugle fra Knoglehuler i Brasilien. E Museo Lundii, 1 (2) [1888], 154.

Woodburne, M.O., Goin, F.J., Bond, M., Carlini, A.A., Gelfo, J.N., López, G.M., Iglesias, A. \& Zimicz, A.N. (2014a) Paleogene Land Mammal Faunas of South America; a Response to Global Climatic Changes and Indigenous Floral Diversity. Journal of Mammalian Evolution, 21 (1), 1-73.

Woodburne, M.O., Goin, F.J., Raigemborn, M.S., Heizler, M., Gelfo, J N. \& Oliveira, E.V. (2014b) Revised timing of the South American early Paleogene land mammal ages. Journal of South American Earth Sciences, 54, 109-119.

Worthy, T.H., Tennyson, A.J.D., Archer, M. \& Scofield, R.P. (2010) First Record of Palaelodus (Aves: Phoenicopteriformes) from New Zealand. Records of the Australian Museum, 62, 77-88.

Wu, Y.-H., Chiappe, L.M., Bottjer, D.J., Nava, W. \& Martinelli, A.G. (2019) Dental morphology and replacement pattern of Late Cretaceous Brazilian Enantiornithine birds. $79^{\text {th }}$ Annual Meeting of the Society of Vertebrate Paleontology (Abstracts of papers): 221 . 
Wu, Y.-H., Chiappe, L.M., Bottjer, D.J., Nava, W. \& Martinelli, A.G. (2021) Dental replacement in Mesozoic birds: evidence from newly discovered Brazilian enantiornithines. Scientific Reports, 11, 19349, 1-12.

Ximenes, C.L. (2009) Tanques Fossilíferos de Itapipoca, CE: Bebedouros e cemitérios de megafauna pré-histórica. In: Winge, M., Schobbenhaus, C., Souza, C.R.G., Fernandes, A.C.S., Berbert-Born, M., Queiroz, E.T. \& Campos, D.A. (Eds.) (2009) Sítios Geológicos e Paleontológicos do Brasil. Volume II. CPRM, Brasília, pp. 465478.

Xu, X. \& Guo, Y. (2009) The origin and early evolution of feathers: insights from recent paleontological and neontological data. Vertebrata PalAsiatica, 47 (4), 311329.

Yamashita, C. (1997) Anodorhynchus macaws as followers of extinct megafauna: an hypothesis. Ararajuba, 5 (2), 176-182.

Zhang, F., Zhou, Z. \& Dyke, G. (2006) Feathers and 'feather-like' integumentary structures in Liaoning birds and dinosaurs. Geological Journal, 41 (3-4), 395-404.

Zelenkov, N.V. (2013) Cenozoic Phoenicopteriform Birds from Central Asia. Paleontological Journal, 47 (11), 1323-1330. 